\title{
Occurrence of peak lifting actions on a large horizontal cantilevered roof
}

\author{
K. M. Lam*, J. G. Zhao \\ Department of Civil Engineering, University of Hong Kong, \\ Pokfulam Road, Hong Kong, China
}

\begin{abstract}
Wind tunnel tests are performed on a rigid model of a horizontal grandstand roof of model dimensions $780 \mathrm{~mm}$ wide and $150 \mathrm{~mm}$ deep. The objective is to investigate the generation mechanism of wind pressure and peak lifting actions on a large cantilevered roof. The roof model is equipped with 78 pairs of pressure taps covering both roof surfaces. With an electronic pressure scanning system, wind pressures at these 156 taps are measured in a near-simultaneous manner. Time histories of the wind forces on both roof surfaces and of the net wind force on the entire roof are obtained from the simultaneous pressure signals. These force signals are analyzed statistically to investigate the mechanism of peak lift forces. The conditional sampling technique is used to extract the wind pressure pattern on the lower and upper roof surface when a large total uplift is occurring on the roof. It is found that the occurrences of peak lift and peak moments on the roof are connected with large lifting actions on both roof surfaces. Differences between the conditionally sampled pressure pattern and the time-average mean pressure pattern are observed and discussed. A study is made on the over-estimation of net roof pressure if it is assumed to be the worst combination of peak pressures simultaneously occurring on both roof surfaces.
\end{abstract}

Keywords: Cantilevered roof, Wind pressure, Wind forces

*Corresponding author: Dr. K. M. Lam,

Department of Civil Engineering,

The University of Hong Kong, Pokfulam Road, Hong Kong

Fax: (852) 25595337

e-mail:kmlam@hku.hk 


\section{Introduction}

Large cantilevered roofs are used mostly as grandstand roofs but there has been an increasing use of huge canopies overhanging from large building developments in metropolitan cities with a purpose to provide sun shading or to act as a noise barrier to highway noise. Wind effects on these roofs are characterised by large uplifting pressure due to the separation of wind flow at the leading edge. This results in a large uplifting force on the roof as well as a large bending moment at the cantilever point. These lifting actions are the major consideration in the structural design of these roofs.

Unlike enclosed building roofs, the net wind effect on a cantilevered roof is a combination of wind pressures on both roof surfaces. The correlation between peak pressures on the upper and lower surfaces thus affect the net lifting actions on the roof. In addition, the inclination of the roof and the blockage offered by the grandstand also affect the wind load pattern. If the roof structure is not perfectly rigid, the loading problem becomes more complicated with the interaction of dynamic wind action and roof vibration modes. Design information of cantilevered roofs comes primarily from two sources; wind loading codes and ad-hoc wind tunnel test. Most wind loading codes provide information on canopy roofs only and the application of these loading data to large grandstand roofs is questionable. In a wind code, the design roof loads are usually taken at the worst situation of simultaneous occurrence of extreme pressures on both roof surfaces. The peak net force on the roof is thus inevitably overestimated. This problem also exists in many ad-hoc wind tunnel tests in which wind pressures are not measured simultaneously on the upper roof surface and on the lower roof surface.

There have been few wind tunnel investigations on cantilevered roofs. Melbourne [1] summarised the results on a number of tests and suggested an equation for an equivalent static design load distribution of the triangular shape for wind loads on rigid and flexible cantilevered roofs. A previous work by Lam and To [2] suggests that the characteristics of wind flow and wind pressure over a cantilevered roof can be quite different from those of an enclosed building roof. For instance, the conical vortices which occur on a building roof at oblique wind angles [3] are not obviously observed on a cantilevered roof.

The use of electronic pressure scanning equipment enables simultaneous pressure measurements to be made on a large number of points on a wind tunnel model. This kind of measurements carried out on models of enclosed building roofs has proven to be very useful in 
understanding the generation mechanism of roof suctions and uplifts $[3,4]$. In this paper, we shall report our wind tunnel investigation on a grandstand roof model in which simultaneous pressure measurements are made on the upper and lower roof surfaces with an electronic pressure scanning system. The pressure data are analysed with conventional statistical techniques and the conditional sampling technique with an aim to understand the generation mechanism of peak suction and lift on a cantilevered roof. The investigation reported in this paper is limited to a horizontal and rigid roof.

\section{Wind Tunnel Experiment}

A generic model of a grandstand roof was chosen to represent a typical large cantilevered roof. A simple rectangular shape was chosen for the roof and the model dimensions were $780 \mathrm{~mm}$ in width and $150 \mathrm{~mm}$ in depth. The roof model was erected horizontally at a height of $180 \mathrm{~mm}$ above the wind tunnel floor. The geometric length scale was targeted at 1:100. The prototype dimensions of the roof are thus $78 \mathrm{~m}$ by $15 \mathrm{~m}$ at a height of 18 m. This would represent a typical grandstand roof for a medium size stadium. Underneath the roof was a model of a stepped grandstand $135 \mathrm{~mm}$ high. The blockage ratio due to the grandstand alone was about 0.75 .

The present basic study on wind pressure on a cantilevered roof was inspired by an adhoc wind tunnel testing of a medium-size stadium roof in Hong Kong [2]. The actual blockage ratio for that grandstand was 0.70 . The value seems very low when compared to larger stadiums reported in the literature. However, it was found that low grandstand blockage ratios are quite common for small to medium size stadiums in Hong Kong. This may be connected with the sub-tropical climate and the mild wind environment under non-typhoon conditions. Some effects of the grandstand blockage are reported in this paper.

Fig. 1a shows the set up of the roof model and the grandstand model. The roof model was supported by seven 6-mm diameter poles along the rear span of the roof. This represented a typical cantilever type of support but was not strong enough in the model scale to prevent vibration of the roof in the wind tunnel. Two 6-mm diameter poles were added at the front roof span to provide extra stiffness. Some modifications to wind flow over the lower roof surface were unavoidable at regions near these supporting poles.

To enable pressure measurement on both surfaces of the roof, the model was constructed from thin acrylic sheets clad on the upper and lower sides of an acrylic framework 
(Fig. 1b). The framework consisted of a rectangular frame and 13 transverse ribs spanning the depth of the roof. Each rib served as an instrumentation bay on which six pairs of pressure taps, each of diameter $0.8 \mathrm{~mm}$, were drilled on both the upper and lower surfaces (Fig. 1c). The overall thickness of the roof model was $6 \mathrm{~mm}$. A total of 78 pairs of pressure taps were installed over the entire roof on a grid of 13 points $\times 6$ points. The 13 rows of taps were equally spaced along the length, or span, of the roof but the 6 pairs of taps in each row were so arranged that increasing smaller tributary areas were associated with taps nearer to either the front or rear edge of the roof. Fig. 2 shows the arrangement of the pressure taps.

Pressure tubing from the tap locations was led inside the roof model in the hollow space between two instrumental ribs and made to exit at the rear edge of the roof through holes in that part of the rectangular frame. The tubing was collected there at six exit points and then led through six columns to the underside of the wind tunnel floor (Fig. 1b). The tubing has an inner diameter of $1 \mathrm{~mm}$. A same tubing length of $700 \mathrm{~mm}$ was used for all taps. The response characteristics of the tubing showed an amplitude distortion less than $5 \%$ at frequencies up to $60 \mathrm{~Hz}$. The tubing from the 156 surface pressure taps in total, together with that from the total and static pressure ports of a pitot-static tube, was connected to five 32-port electronic pressure scanners (PSI Inc.) mounted underneath the wind tunnel floor. The pressure scanners were controlled by a data acquisition unit (Aeroprobe Inc.) and near-simultaneous pressure measurements were made on all the taps with the pressure scanners at a rate of $100 \mathrm{~Hz}$ per tap. The time signals were recorded for a period of 60 seconds and saved on computer files for later processing. A pitot-static tube was placed at a height of twice the roof height to monitor the wind speed and the static pressure inside the wind tunnel.

The experiments were carried out in the boundary layer wind tunnel of the Department of Civil Engineering, The University of Hong Kong. The wind tunnel was of the recirculating type with a $12 \mathrm{~m}$ long working section of $3 \mathrm{~m}$ wide by $1.8 \mathrm{~m}$ high. Spires and roughness elements were used to simulate at the centre of the turntable wind conditions of the general type terrain in the Hong Kong Wind Code at a scale of 1:100 [5, 6]. This terrain type, which specifies a power law exponent of 0.19 for the mean wind speed profile, corresponds to somewhere between the open land and suburban terrain in other codes. The mean wind speed profile measured with a hot-wire is shown in Fig. 3a in the log-law format. The value of $z_{o}$ was found to be $1.1 \mathrm{~mm}$ in the wind tunnel or $0.11 \mathrm{~m}$ full-scale. The measured profile of turbulence intensity was shown in Fig. 3b. It was found to decrease with height and had a value of 0.12 at the roof height. Fig. 4 shows the turbulence spectrum measured at a height of $500 \mathrm{~mm}$ and it 
suggested a value of the integral scale of turbulence at $270 \mathrm{~mm}$ in the wind tunnel or $27 \mathrm{~m}$ in full scale. During the tests, the wind tunnel was run with a wind speed of about $10 \mathrm{~m} / \mathrm{s}$ at the roof height and this speed corresponded to the extreme wind conditions in Hong Kong at a velocity ratio of 1:4. The time ratio is thus 1:25.

The sampling rate and period of pressure measurement at $100 \mathrm{~Hz}$ and $60 \mathrm{~s}$, respectively, appeared too low to allow an accurate estimation of peak pressures. The low values used were due to the limitation of the data acquisition unit in handling the large number of pressure taps. For strong wind simulation, the time scale of the wind tunnel tests was 1:25. The corresponding full-scale sampling period was $25 \mathrm{~min}$. As described later, this period was further divided into two sub-periods for peak pressure estimation. The 12.5-min. length of the sub-period was just below the commonly accepted 15-min. to 1-hour period for statistically confident wind measurement. The full-scale sampling rate was $4 \mathrm{~Hz}$ which should be high enough for resolving gust effect on small areas. For peak pressure measurements on building models, criteria of minimum sampling rate and tubing response frequency have been proposed at $6 U_{h} / B$ and $2 U_{h} / B$, respectively [7]. $U_{h}$ is the mean wind speed at the roof of the building model and $B$ is the smallest model width. Adopting these criteria with the roof depth as $B$, the required minimum values were $400 \mathrm{~Hz}$ and $133 \mathrm{~Hz}$ approximately. The values used in the present wind tunnel experiments were 2 to 4 times lower. It was thus estimated that the peak pressures might be underestimated by up to $10 \%$. The main focus of this paper was on the mechanism of peak loading actions on the roof rather than to obtain numerical values of pressure coefficients. The possible errors in peak pressure estimation were not detrimental enough to affect the phenomenal interpretation of the results.

\section{Results and discussion}

The record of wind pressure at each tap location is analyzed to obtain the mean value, $\bar{p}$, and peak values, $\hat{p}, \breve{p}$. The standard deviation $\sigma_{p}$ of the pressure fluctuations is also calculated. These pressure values are presented in the form of pressure coefficients using the mean wind speed $U_{h}$ at the roof height as the reference velocity, e.g.:

$$
\bar{C}_{p}=\frac{\bar{p}}{1 / 2 \rho U_{h}^{2}}
$$

Time signals of wind forces on different roof surfaces are obtained from an integration of the near-simultaneous record of wind pressures over the corresponding surfaces. The mean, 
standard deviation and peak values of wind forces are calculated from the time signals. They are presented as force coefficients using the one-sided surface area $A$ of the roof as the reference area. The peak force coefficient for the upper roof surface, for instant, is:

$$
\hat{C}_{F_{\text {upper }}}=\frac{\hat{F}_{\text {upper }}}{1 / 2 \rho U_{h}^{2} A}
$$

The convention for positive roof pressure, wind force and the corresponding pressure and force coefficients, on either the upper or lower roof surface is always from the air side onto the roof surface. The net wind-induced pressure and force on the roof due to the combined effect of the upper and lower roof surfaces is defined as positive in the vertically downward direction. The relationships are like this example:

$$
p_{\text {net }}=p_{\text {upper }}-p_{\text {lower }}
$$

The wind incidence angle $\alpha$ is measured from the direction coming directly from the front of the cantilevered roof at $\alpha=0^{\circ}$.

\subsection{Pressure patterns on roof surfaces}

Distributions of time-averaged mean wind pressure coefficient on the upper and lower surfaces of the roof are shown in Fig. 5 at wind incidence angles of $\alpha=0^{\circ}, 20^{\circ}, 30^{\circ}$, $60^{\circ}, 90^{\circ}, 120^{\circ}, 150^{\circ}$ and $180^{\circ}$. Distributions of the net mean wind pressure coefficient on the roof as a whole are also shown in the figure. Their patterns are similar to those of the previous ad-hoc study made on a real stadium roof in the same wind tunnel laboratory [2]. An additional finding is observed here in Fig. 5 that the net wind pressure is contributed more significantly by the upper roof pressure than by the lower roof pressure, both in magnitude and in distribution patterns. When the wind is coming from the front of the roof, $\alpha<90^{\circ}$, the pattern and level of the wind pressure on the lower roof surface do not change much with the wind angle. The upper roof pressure pattern is however found to change significantly with the wind angle and the net roof pressure shares a very similar pattern as the upper roof pressure.

At $\alpha=0^{\circ}$, the entire upper roof surface is under suction with very high suction pressure, that is negative pressure coefficient, at the windward edge. This is caused by separation of wind flow along the windward edge. Highest suction is found at very local areas at the two windward corners. On the lower roof surface, the pressure is mostly 
positive, that is acting in the upward direction. Highest positive pressure is found at the frontal part of the roof and the pressure level decreases in going downwind to the rear edge of the roof. Under the combined pressure effect on both surfaces, the time-averaged net pressure coefficient pattern shows a high suction pressure region along the front edge of the roof. A value of $\bar{C}_{p, n e t} \cong-1.8$ is found along the front roof edge. Out of this, the upper roof pressure contributes a value of about -1.0. At the two upwind corners, even higher suction pressure at $\bar{C}_{p, n e t}<-2$ is found and this is almost contributed totally by the upper roof pressure.

When the wind is at a slightly oblique angle, at $\alpha=20^{\circ}$, the pressure coefficient pattern on the lower surface remains very similar to that at $0^{\circ}$ incidence but on the upper roof surface, there is an obvious shift of the pressure coefficient pattern with the wind incidence angle. Furthermore, a localized region of very high suction is found at the upwind corner. The region appears to be consisting of two V-shaped stripes of high suction. This resembles the effect due to the conical vortices found on a building roof at oblique wind incidence [3]. The distribution pattern of the net roof pressure coefficient follows mostly that of the upper roof pressure coefficient. The largest levels of negative pressure coefficient are similar to those at $\alpha=0^{\circ}$ and they are contributed mostly by the pressure coefficient on the upper roof surface.

The distribution patterns of pressure coefficients at $\alpha=30^{\circ}$ are similar to those at $\alpha$ $=20^{\circ}$. The distribution of net pressure coefficients in Fig. $5 \mathrm{c}$ shows a region of largest negative net pressure along the middle part of the windward roof span. The mean net pressure coefficients there have values of $\bar{C}_{p, n e t}<-1.6$. This magnitude is slightly lower than that at $\alpha=20^{\circ}$ and is mainly due to a lower suction level on the upper surface.

At $\alpha=60^{\circ}$, the mean net suction pressure coefficients on the roof have much lower magnitudes. There only remains a narrow strip of $\bar{C}_{p, n e t}<-0.8$ along the front edge over the middle half span. The pressure values on the lower roof surface, however, remain very similar to those found at the earlier wind incidence angles. On the upper surface, the high suction region at the upwind corner is no longer found. Instead, there is a small patch of slightly positive pressure centered at the upwind $1 / 4$ span of the roof. This suggests that some flow reattachment may occur there.

At $\alpha=90^{\circ}$, wind is blowing along the roof span. Small values of positive pressure or suction pressure are found on either roof surfaces. Disturbed wind flow around the two front 
supporting poles is responsible for the two localized regions of negative pressure observed on the lower roof surface.

When $\alpha>90^{\circ}$, positive upward pressure on the lower roof surface along the windward rear roof edge is of lower values than the situation when wind comes from the front. Because of the blockage effect of the grandstand, wind passing underneath the roof now flows through the clearance at the back of the sloping grandstand with a higher velocity but continues to decelerate over the grandstand. The pressure pattern on the upper surface has slightly lower suction values and exhibit smoother distribution, when compared to the situation of wind incidence from the front. The high suction pressure regions at the upwind corners are not observed.

Fig. 6 shows the coefficient of net pressure standard deviation on the roof. When comparing this pattern at $\alpha=0^{\circ}$ with that of the mean pressure coefficient (Fig. 5a), an obvious difference is observed. While the latter shows a separation bubble along the front edge of the roof with almost parallel contour lines of mean pressure, the former pattern reveals two regions of high pressure fluctuations at the two downwind corners of the mean separation bubble. This may imply that the shape of the separation bubble changes violently with time at these two locations or that the instantaneous separation bubble smears to either side in two preferred orientations. This observation will be further supported by the later conditional sampling results. At $\alpha=30^{\circ}$, the pattern of pressure fluctuations at the upwind corner reveals the possible occurrence of conical vortices in a more evident manner than the mean pressure pattern. When wind blows from the rear of the roof, the net pressure is under smaller degree of fluctuations than the situation of wind incidence from the front. A similar pattern as $\alpha=0^{\circ}$ is observed at $\alpha=180^{\circ}$ where two regions of high pressure fluctuations are found at the corners of the mean separation bubble.

\subsection{Peak roof pressures}

From the time history of pressure signals, an estimation of peak pressures is made at each tap location following the method of Peterka [8]. The time history is divided into two sub-periods and the maximum (and minimum) values in the two sub-periods are averaged and then increased by $7 \%$ to obtain an estimate of the peak maximum and peak minimum pressure. Although this is not a rigorous procedure for peak pressure estimation, it may be adequate for the purpose of comparison study. Fig. 7 shows the distribution of the peak 
maximum and peak minimum pressure coefficients on the roof. These peak values are estimated directly from the time history of the net pressure coefficient signals, $C_{p, n e t}(t)$ and represent the true peak net pressure coefficients on the roof:

$$
C_{p, \text { net }}(t)=C_{p, \text { upper }}(t)-C_{p, \text { lower }}(t)
$$

At $\alpha=0^{\circ}$, the peak minimum pressure coefficient on the roof along the windward span of the roof goes as high as $\breve{C}_{p, n e t}<-3.6$. The values are about double the mean net pressure coefficient values in Fig. 5. As for the peak maximum pressure, suction pressure at $\hat{C}_{p, n e t}<-0.6$ is still found at the front part of the roof but towards the rear span, low magnitude net pressure acting downwards are found. Roughly the same values of highest peak maximum and minimum pressure coefficient are found along the frontal roof span at $\alpha=30^{\circ}$ as those at $\alpha=0^{\circ}$. At $\alpha=60^{\circ}$, the highest peak minimum pressure at $\breve{C}_{p, \text { net }}<-2.0$ is found at the frontal roof span and this is more than double the mean pressure coefficient value at $\bar{C}_{p, n e t}<-0.8$ in Fig. 5. The other extreme value of peak maximum pressure coefficient $\hat{C}_{p \text {,net }}$ is slightly positive over most of the roof surface. The two regions of high positive values for peak maximum pressure coefficients at the frontal span are believed to be a result of flow around the two supporting poles on the lower surface of the roof. At $\alpha=90^{\circ}$, the values of peak maximum and peak minimum pressure coefficient have nearly the same magnitudes but with opposite signs.

In situations of wind loading codes and wind tunnel tests with pressure not sampled simultaneously on both roof surfaces, the peak pressures and suctions on the two surfaces are obtained separately on the two roof surfaces. A conservative estimation of the peak net pressure coefficient can only be made by assuming the worst situation of peak pressures occurring at the same instant on both surfaces. The conservative estimates are:

$$
\begin{aligned}
& \hat{C}_{p, \text { net(est.) }}=\hat{C}_{p, \text { upper }}-\breve{C}_{p, \text { lower }} \\
& \breve{C}_{p, \text { net(est.) }}=\breve{C}_{p, \text { upper }}-\hat{C}_{p, \text { lower }}
\end{aligned}
$$

Fig. 8 shows the amount of overestimation of the peak minimum and peak maximum net pressure coefficient on the roof when they are estimated from (5). The overestimation is calculated in terms of the level difference in pressure coefficients between the true peak values and the estimated peak values, that is, $\left(\breve{C}_{p, \text { net(est. })}-\breve{C}_{p, \text { net }}\right)$ for the peak minimum pressure coefficient, and $\left(\hat{C}_{p, \text { net (est. })}-\hat{C}_{p, n e t}\right)$ for the peak maximum pressure coefficient. 
For the peak minimum pressure, Fig. 8a shows that the amounts of overestimation are relatively uniform over the roof and average about a value of 0.4 to 0.6 in terms of $C_{p, n e t}$. At the edge zones where the peak suction values are high, the overestimation is smaller than the average overestimation value. This suggest that when a peak suction is occurring, there is strong correlation between the pressures on the two roof surfaces, thus bringing the assumed situation of simultaneous occurrence of peak pressures on the two roof surfaces closer to the real situation. For the peak maximum pressure, the amounts of overestimation have a larger average value of about 0.6 (Fig. 8b).

Statistically, the true values of peak net pressure can be computed from the mean and standard deviation values of pressure on the two surfaces if the cross correlation coefficient $R_{u l}$ between the upper and lower roof surface pressure signals is known or measured:

$$
\begin{aligned}
& \hat{p}_{\text {net }}, \text { or } \breve{p}_{\text {net }} \\
& =\left(\bar{p}_{\text {upper }}-\bar{p}_{\text {lower }}\right) \pm g \sqrt{{\sigma_{p, \text { upper }}{ }^{2}-2 R_{u l} \sigma_{p, \text { upper }} \sigma_{p, \text { lower }}+\sigma_{p, \text { lower }}^{2}}^{2}}
\end{aligned}
$$

In this expression, a same value of peak factor $g$ is assumed for peak pressure on both surfaces but this may not be true from the present data:

$$
\hat{p}=\bar{p}+g \sigma_{p}
$$

The validity of (6) is thus not tested on the present data. Instead, the cross correlation between the wind forces on the two roof surfaces is studied.

\subsection{Wind forces on roof surfaces}

Fig. 9 shows an example time slice of the wind force signals on the upper and lower roof surfaces and on the roof as a whole. They are obtained from integration of the nearsimultaneous pressure signals at $\alpha=30^{\circ}$. All three force coefficients represent uplifting effect on the roof. The force on the upper roof surface contributed more to the total net roof force than the lower roof surface force.

Coefficients of mean, peak values and standard deviations of the roof forces are shown in Fig. 10 at different wind angles. Most of the mean force coefficients show the effect of uplifting load on the roof except the lower surface force coefficient at $\alpha=100^{\circ}$ to $160^{\circ}$. The magnitudes of downward loading in these exceptions are small and do not produce a significant overall effect. For the peak values, the upper roof force sometimes exerts a small downward load at wind angles between $\alpha=60^{\circ}$ to $130^{\circ}$ while the lower roof 
force sometimes produces a large upward lift at $\alpha>50^{\circ}$. The standard deviation of the force has nearly constant values for the two roof surfaces but for the net force, smaller values of fluctuations are observed around $\alpha=90^{\circ}$ where the mean net force is close to zero.

The previous results on pressure distribution suggest that there appears to be some connection between the wind pressure generation mechanisms on the two roof surfaces. This should be reflected in the joint statistics between the wind forces on the two roof surfaces. Fig. 11 shows the joint probability density function (joint-pdf) between the two surface force signals. The joint-pdf is the probability of the forces having a particular combination of values at the same time:

$$
p\left(C_{F, \text { lower }}, C_{F, \text { upper }}\right)=\operatorname{Prob}\left\{C_{F, \text { lower }}(t)=C_{F, \text { lower }}, C_{F, \text { upper }}(t)=C_{F, \text { upper }}\right\}
$$

At $\alpha=90^{\circ}$, the three-dimensional hill of the joint-pdf is a narrow steep cliff elongated along the diagonal of increasing values of both forces. This means that the lifting actions on both surfaces rises and falls together. A suction effect on the upper surface is always accompanied by a suction load on the lower surface. As a result, the total net force on the roof is always very small. This may be because the flow is similar to that over a flat plate where the roof plays a small role in modifying the incoming wind. Fluctuations in the vertical wind load are then caused largely by the turbulence in the approaching wind. At $\alpha=60^{\circ}$, the joint-pdf hill is broader but is still elongated along the same diagonal. However around the outer rim of the broad hill shaped distribution, there exist many undulations. This implies that the wind force actions on the two surfaces tend to rise and fall together only during the time when both forces are around their mean values. At peak values of the forces, their relationship is more random. At $\alpha=0^{\circ}$ and $30^{\circ}$, the joint-pdf is much broader with more undulations around the rim.

Fig. 12 shows the cross correlation function between the total net roof force with either the upper or lower roof surface force:

$$
R_{F_{1} F_{2}}(\tau)=\frac{1}{\sigma_{F_{1}} \sigma_{F_{2}}} \frac{1}{T} \int_{0}^{T} F_{1}(t) F_{2}(t+\tau) d t
$$

It is evident that at wind incidence angles from the front of the roof, the occurrence of a large total lift on the roof is connected with the occurrence of a large lifting action on the upper surface as well as the occurrence of a large upward force on the lower surface. However, the connection of the total net force with the upper surface force is always closer than with the lower surface force. At $\alpha=0^{\circ}$ and $30^{\circ}$, the cross-correlation coefficients for the former reaches as high as 0.6 and for the latter is about 0.3 . The highest correlation 
coefficients drop to lower values at $\alpha=60^{\circ}$. At $\alpha=90^{\circ}$, there is little correlation of the total net force with either surface force.

\subsection{Conditional sampling of wind forces}

To understand the generation mechanism of peak lift force on the roof, the conditional sampling technique is employed. An occurrence of peak lift on the roof is registered when the total net roof force is lower than its mean value by three times its standard deviation (Fig. 9) This triggers a conditional sampling of the three wind force signals with pre-trigger and post-trigger delay time intervals with respect to the instants of the highest negative peak below the trigger level. Within the total record length of 60 seconds, there are about 5 to 20 these occurrences of peak total lift on the roof. One of these occurrences can be seen in Fig. 9. The samples of force signals triggered by these occurrences are ensemble averaged to represent the typical pattern when a peak lift is occurring. Fig. 13 shows the resulting force coefficient signals after conditional sampling. The lack of a repetitive pattern in the self-sampled total lift force signal suggests that an occurrence of peak total lift appears to be an isolated event. At $\alpha=0^{\circ}$ and $30^{\circ}$, the results suggest that the generation of peak total lift on the roof is contributed by simultaneous occurrence of large lifting forces on both roof surfaces. At $\alpha=60^{\circ}$, the contribution of the lower roof surface force to the peak total roof lift is not obvious. At $\alpha=90^{\circ}$, the occurrences of peak lift on different roof surfaces are loosely inter-related.

\subsection{Conditional sampling and correlation of wind pressure patterns}

In addition to the contributions from the wind forces on the two roof surfaces, it is important to investigate whether there is a particular pattern of wind pressure distribution connected with the occurrence of a peak total lift on the roof. Using the same trigger criteria as for the wind forces, the wind pressures on the roof surfaces are conditionally sampled at the occurrence instants of total peak lift. Fig. 14 shows the distribution patterns of the conditionally sampled net roof pressure coefficient and the corresponding pressure coefficients on the two roof surfaces. When compared with the mean pressure coefficient contours in Fig. 5, the conditionally sampled pressure coefficients have much larger magnitudes, especially on the upper roof surfaces. This reinforces the observation that the 
upper roof surface contributes more to the occurrence of peak overall lift on the roof. On the lower roof surface, the patterns of the mean and conditionally sampled pressure coefficient contours are similar. On the upper roof surface, the two sets of contours display obviously different patterns. As a result, the pattern of the conditionally sampled net pressure coefficient distribution is different from that of the mean net pressure coefficient.

At $\alpha=0^{\circ}$, Fig. 14a shows that during the occurrence of a peak total roof lift, the front half of the lower roof surface is under an upward pressure at $C_{p \text {,lower }}>0.4$ while in the time-averaged sense, the same area is under a slightly lower mean pressure roughly at $\bar{C}_{p, \text { lower }}>0.35$ (Fig. 5a). The distribution patterns under the two situations are very similar. On the upper surface, however, the patterns shown in Fig. 14a and in Fig. 5a are very different. While the mean pressure coefficient pattern suggests the existence of a large and uniform separation bubble spanning almost the entire span of the roof along the front edge, the conditional sampling result tends to suggest separation bubbles of shorter spans. Following the contours of $C_{p, \text { upper }}<-1.2$ in Fig. 14a, these separations are more localized but associated with higher suction levels. They are found to extend a farther distance into the depth of the roof and appear to have a preferred orientation towards the downwind corners of the mean separation bubble along a laterally outward oblique direction. This observation has been suggested in the earlier results of distribution of pressure fluctuations in Fig. 6.

As a result of the occurrence of strong separation on the upper roof surface and the possible existence of intense localized separation bubbles, the front half of the upper roof is under a much higher suction at $C_{p \text {,upper }}<-1.0$ in Fig. 14a. For the mean pressure in Fig. 5a, the same area is under a suction of $\bar{C}_{p, \text { upper }}<-0.6$. It is again evident that the upper surface contributes mostly to the net wind effect on the roof. This is the case of the mean lifting effect and for the occurrence of peak lifting action, the contribution from the upper surface is even more significant. Accordingly, the distribution pattern of the net roof pressure in Fig. $14 \mathrm{a}$ resembles mostly the pattern of the upper surface pressure. There are obvious protrusions of the high suction zone laterally into the two downwind corners of the mean separation bubble.

Similarly at $\alpha=30^{\circ}$, little differences are observed on the conditionally sampled and mean pressure patterns on the lower surface. On the upper surface, however, Fig. 14b shows that during the occurrence of peak total roof lift, the pressure coefficient contours of high negative pressure are aligned at a more oblique angle than those of the mean pressure coefficient (Fig. 5c). The contours also form a closed region which suggests some form of 
coherent flow separation. On an enclosed building roof, the conditionally sampled suction pattern has been found to reveal the conical vortices and their orientation more clearly than the mean pressure pattern [3]. It is not certain of the physical phenomena connected with the present observation in Fig. 14b but it is clear that the region of coherent separation in the conditionally sampled pressure on the upper roof results in much high suction levels. In the mean pressure pattern (Fig. $5 \mathrm{c}$ ), the front half of the roof upper surface is under suction of $\bar{C}_{p, \text { upper }}<-0.4$; while during the occurrence of a peak total lift, the same area is under higher suction of $C_{p, \text { upper }}<-0.6$. This is in sharp contrast with the observation on the lower surface where little differences are found.

At the more oblique wind incidence from the front at $\alpha=60^{\circ}$, even a higher rise is observed for the conditionally sampled upper roof surface pressure as compared with the mean pressure. For the front half surface, the rise is almost double from $\bar{C}_{p, u p p e r}<-0.2$ to $C_{p \text {,upper }}<-0.4$ in Fig. 14c. However, on the lower surface, it is surprising to find that after conditional sampling, the pressure levels are even lower than the mean pressure values. This may reflect the lack of correlation of the lower surface effect with the total wind effect on the roof, as suggested earlier in Fig. 13, but it is felt that other unknown causes may need to be sought for this strange observation.

When wind blows along the roof span at $\alpha=90^{\circ}$, the conditionally sampled pressure pattern on the upper surface reveals flow separation on the windward edge followed by flow reattachment (Fig. 14d). This is not observed on the time-averaged pressure pattern in Fig. 5e. The implication is that flow separation at the roof edge only occurs intermittently and this is always coincident with the occurrence of a large total roof lift at this wind angle.

At wind angles where wind blows from the rear of the roof, less obvious differences are observed between the patterns of the conditional sampled pressure coefficient on the upper roof in Fig. 14 and those of the mean pressure coefficient in Fig. 5. There are still differences in magnitudes of the negative pressure coefficients between the two sets of data in Figs. 5 and 14. The levels of magnitude differences are similar to those found at $\alpha<90^{\circ}$. At $\alpha=180^{\circ}$, localized separation bubbles similar to those at $\alpha=0^{\circ}$ are observed.

In addition to the uplift, the overturning moment about the rear edge of the roof is another principal wind load to be considered in the structural support of the cantilevered roof. It is thus worth repeating the investigation with regard to the occurrence of peak moment. Using the same trigger criteria but on the roof moment signal instead of the force 
signal, the distribution of wind pressure coefficient on the roof surfaces were conditionally sampled and the results shown in Fig. 15 for $\alpha=0^{\circ}$ to $90^{\circ}$. The same basic patterns are observed as in Fig. 14 but the sampled suction levels on the upper roof surface are even higher, though only slightly. This is especially so on the frontal part of the roof. This supports the proposal that some forms of devices which interfere with flow separation at the roof front would reduce the overturning moment in addition to the uplift [9].

The preceding conditional sampling results suggest that the mean separation bubble revealed on the upper roof surface by the distribution of mean pressure coefficient (Fig. 5) may not represent an instantaneous pressure pattern when a peak total lift is occurring. To investigate whether coherent flow separation occurs over the roof span statistically at all, spatial cross correlation analysis was performed on the net roof pressure signals. Fig. 16 shows the contours of correlation coefficient (at zero time lag) of the net roof pressure with the reference net roof pressure taken at the most frontal pair of pressure taps at the mid-span.

Even at $\alpha=0^{\circ}$, the correlation coefficient drops off rapidly with the spatial separation from the reference point. The correlation coefficient can maintain a value above $R_{p p}(\tau=0)=0.5$ only on a small area around the reference point. The area over which the correlation coefficient stays above a level of $R_{p p}(\tau=0)=0.3$ only has a size of about $1 / 4$ the roof span by $1 / 3$ the roof depth. The pattern of mean net roof pressure in Fig. 5a shows a region of uniformly high mean negative pressure covering nearly the entire span of the roof along the front edge. However, the low degree of correlation between pressure signals at different locations along the roof span found in Fig. 16 does not support the occurrence of a flow structure or flow event of that large scale. The apparently large separation bubble in Fig. 5a results from the long time averaging of the effects of many localized flow separations of smaller spatial scales. Some of these smaller-scale separations are much more intense than average and result in very high suctions on the roof surface. The strongest of these localized separations are believed to be responsible for the generation of peak total lift on the roof (Fig. 14a).

At $\alpha=30^{\circ}$ and $60^{\circ}$, the region of significant correlation level is smaller in size than that at $\alpha=0^{\circ}$ and its shape is aligned with the oblique wind incidence. At the latter wind angle, a downwind lobe is found extending along the wind direction. At $\alpha=90^{\circ}$, the pattern is more dispatched.

\subsection{Effect of grandstand blockage}


The effect of grandstand blockage is not the main focus of this investigation but it may be useful to include some data for the extreme cases of no blockage and complete blockage. The data shown here in Fig. 17 are the mean pressure coefficients on the roof surfaces at $\alpha=0^{\circ}$ only.

At $100 \%$ blockage, positive pressure coefficients are found on the entire lower roof surface. Very high upward pressure up to $\bar{C}_{p, \text { lower }}>1.2$ is found along the middle depth of the lower roof surface (Fig. 17a). This is because wind entering in the grandstand is trapped here leading to a strong stagnation effect. The pressure pattern on the upper roof surface has some obvious differences from that in Fig. 5a. The magnitudes of negative pressure found along the windward span are higher. This can be explained by a larger amount of wind flow over the upper roof surface. There are two localized regions of higher suction, each centered at a location about 1/6 roof span distance from either side edge of the roof and about 1/3 roof depth distance from the windward span. These two localized region of intense flow separation are similar to the localized high suction regions in the conditionally sampled pressure pattern in Fig. 14a. The combined effect of both surfaces leads to the net pressure coefficient pattern in Fig. 17a where high upward net pressure is found in a strip region along the central part of the span near the middle depth of the roof in addition to the high upward pressure region along the windward span.

When there is the cantilevered roof alone without the grandstand, Fig. 17b shows smoother contours of constant pressure coefficients on both roof surfaces. Negative pressure is found on the upper roof and is caused primarily by wind separation along the windward span. The levels are lower than the situation with a grandstand because more wind flow now passes underneath the roof. This also seems to result in a more steady flow over the upper roof surface leading to a smoother pressure pattern. Wind pressure has small magnitudes over a large central part of the lower roof surface. Disturbed flow regions around the supporting columns and poles lead to some local pressure patterns. A significant scale of wind separation over the lower roof surface is not evident along the windward span. It appears that even though there is no grandstand blockage, the flow underneath the roof is somewhat restrained by the ground as well. A large part of the roof is under net wind coefficients of magnitudes below $\bar{C}_{p, n e t}=-0.6$. The highest levels of largest upward net pressure along the windward roof span only reach values near $\bar{C}_{p, \text { net }}=-1.2$. 


\section{Conclusions}

A rigid model of a horizontal cantilevered grandstand roof was tested in the wind tunnel. Wind pressures were measured at 156 taps covering both surfaces of the roof in a near-simultaneous manner with electronic pressure scanners. Time histories of wind forces on the whole roof and on the lower and upper roof surfaces were obtained from the pressure data. The data were analyzed by conventional statistical methods and the conditional sampling technique to explore the generation mechanism of overall and peak wind lift on the roof.

It was found that the horizontal roof is under a mean lifting action at most wind incidence angles. This is under the combined effects from both roof surfaces with the upper roof surface contributing more lifting effect. Correlation and conditional sampling analysis showed that the occurrence of peak total roof lift is connected with simultaneous large lifting actions on both roof surfaces, with a more significant contribution from the upper surface.

An evaluation was made on the degree of overestimation in peak net pressure if separately obtained peak pressure values on the lower and upper roof surfaces are combined to form the worst situation. The degree of overestimation is around 0.5 in terms of pressure coefficients and the relative degree of overestimation is smaller at regions of high suction. This is consistent with the suggestion that the occurrence of peak pressure on the roof is connected with nearly simultaneous pressure effects on the two roof surfaces.

After conditional sampling, the distribution of roof pressure at the instants of occurrence of peak total roof force and roof moment revealed that the suction pressure on the upper surfaces is about 1.5 times to double the mean value. At wind incidence from the front of the roof, very high suction is found on the front edge of the roof. The pressure on the lower roof surface at the instant of peak roof lift is higher than the mean value by a smaller ratio. While the patterns of the conditionally sampled pressure and the mean pressure are similar on the lower roof surface, they show some significantly different features on the upper roof surface. At wind incidence from the front, there seems to be, instantaneously, localized wind separation regions along the front edge of the upper roof surface instead of a large uniform separation bubble spanning the entire front edge of the roof as revealed by the 
mean pressure pattern. These localized separation zones appear to penetrate more deeply into the roof depth along two laterally oblique directions.

\section{Acknowledgement}

The investigation is supported by a research grant awarded by the Research Grants Council of Hong Kong. The authors wish to thank Prof. H. Kawai (Kyoto University) for his valuable advice on the work. 


\section{References}

[1] W.H. Melbourne, The response of large roofs to wind action, J. Wind Eng. Ind. Aerodyn. 54/55 (1995), 325-336.

[2] K.M. Lam, A.P. To, Generation of wind loads on a horizontal grandstand roof of large aspect ratio, J. Wind Eng. Ind. Aerodyn. 54/55 (1995), 345-357.

[3] H. Kawai, G. Nishimura, Characteristics of fluctuating suction and conical vortices on a flat roof in oblique flow, J. Wind Eng. Ind. Aerodyn., 60 (1996), 211-225.

[4] N. Hosoya, J.E. Cermak, S.M. Dodge, Area-averaged pressure fluctuations on surfaces at roof corners and gable peaks, Proc. $10^{\text {th }}$ Int. Sym on Wind Eng., Copenhagen, 1999, 1779-1785.

[5] E.C.C. Choi, Wind loading in Hong Kong, Commentary on the Code of Practice on wind effects, Hong Kong, 1983.

[6] Building Development Department, Hong Kong, Code of Practice on Wind Effects, Hong Kong - 1983, 1983.

[7] J.D. Holmes, Effect of frequency response on peak pressure measurements. J. Wind Eng. Ind. Aerodyn., 17 (1984), 1-9.

[8] J.A. Peterka, Selection of local peak pressure coefficients for wind tunnel studies of buildings, Wind Eng. Ind. Aerodyn., 13 (1983), 477-488.

[9] W.H. Melbourne, J.C.K. Cheung, Reducing the wind loading on large cantilevered roofs, J. Wind Eng. Ind. Aerodyn. 28 (1988), 410-410. 
Lam \& Zhao, Fig. 1

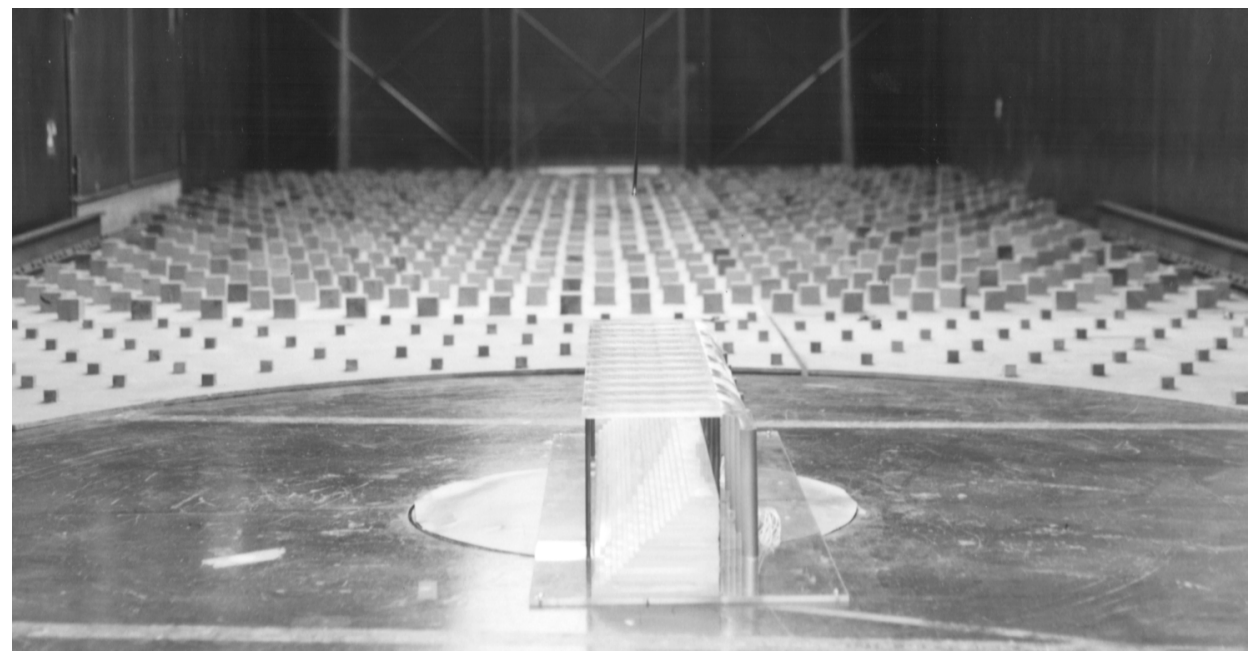

(a)

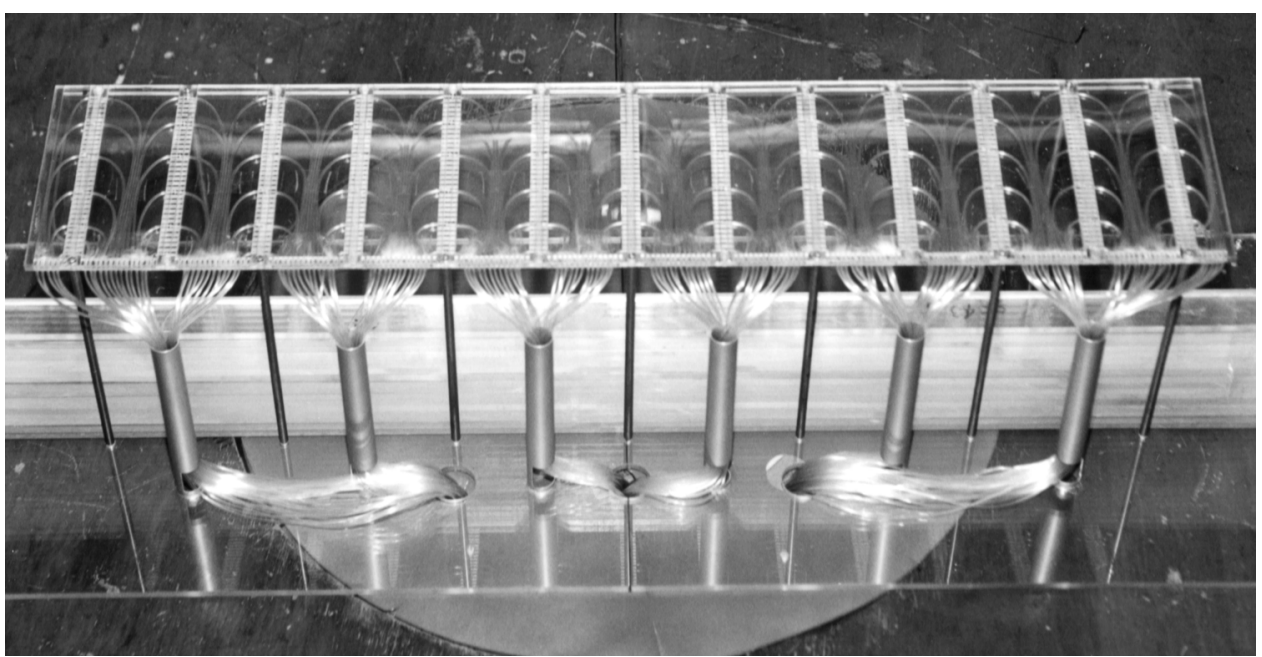

(b)

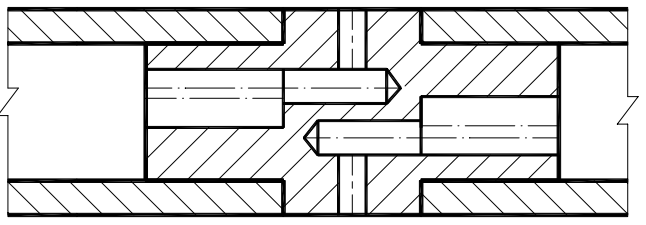

(c)

Fig. 1. Wind tunnel model of cantilevered roof and grandstand. (a) Set up in wind tunnel. (b) Construction of roof model. (c) Section through instrumental rib showing installation of pressure taps. 


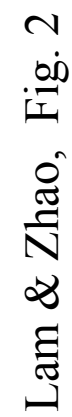
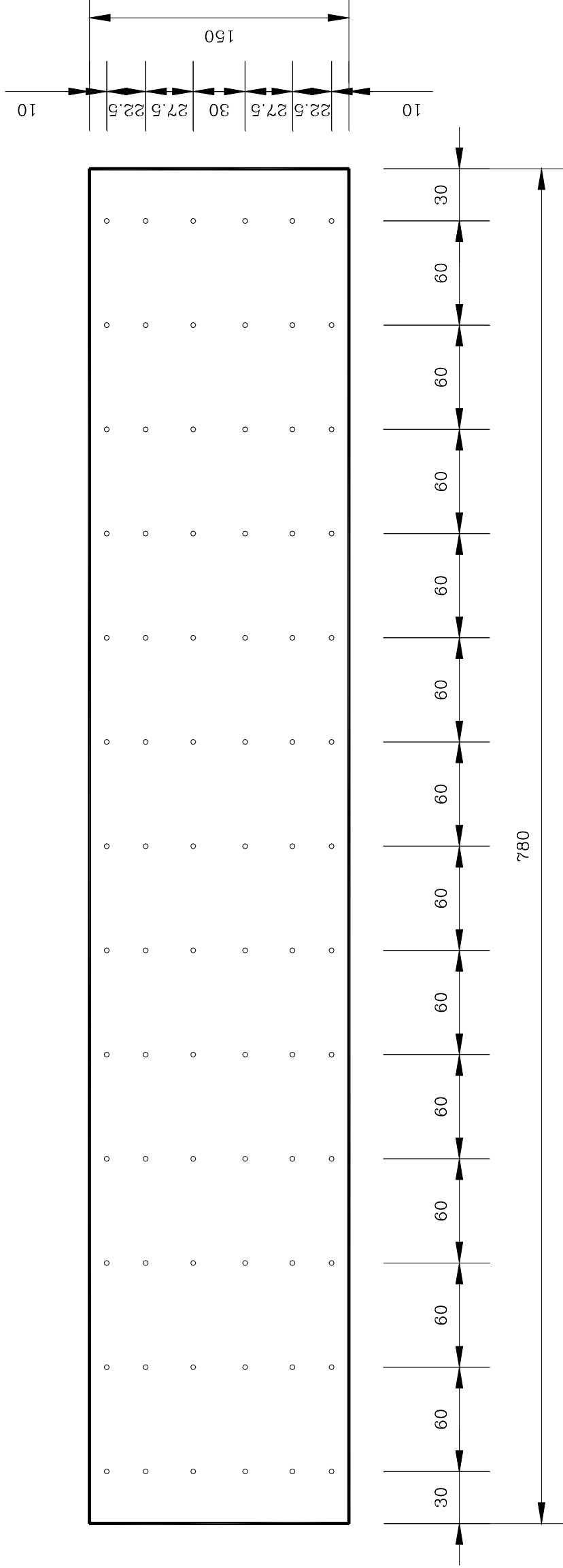

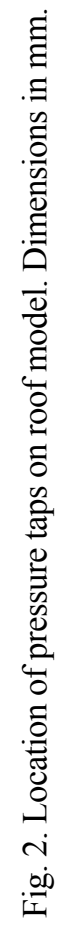



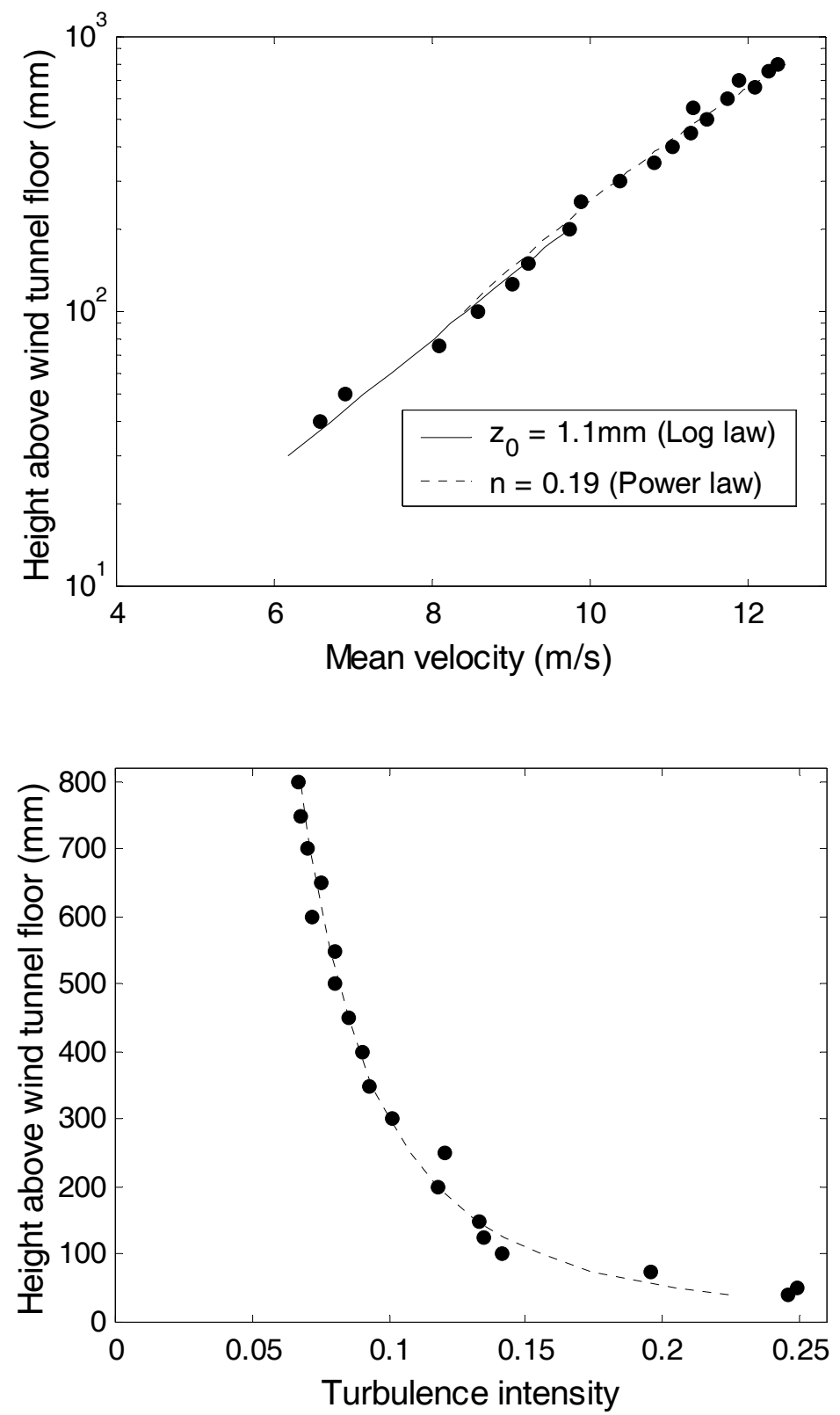

Fig. 3. Simulated wind conditions in wind tunnel. (a) mean wind speed profile; (b) turbulence intensity profile with power law fit. 
Lam \& Zhao, Fig. 4

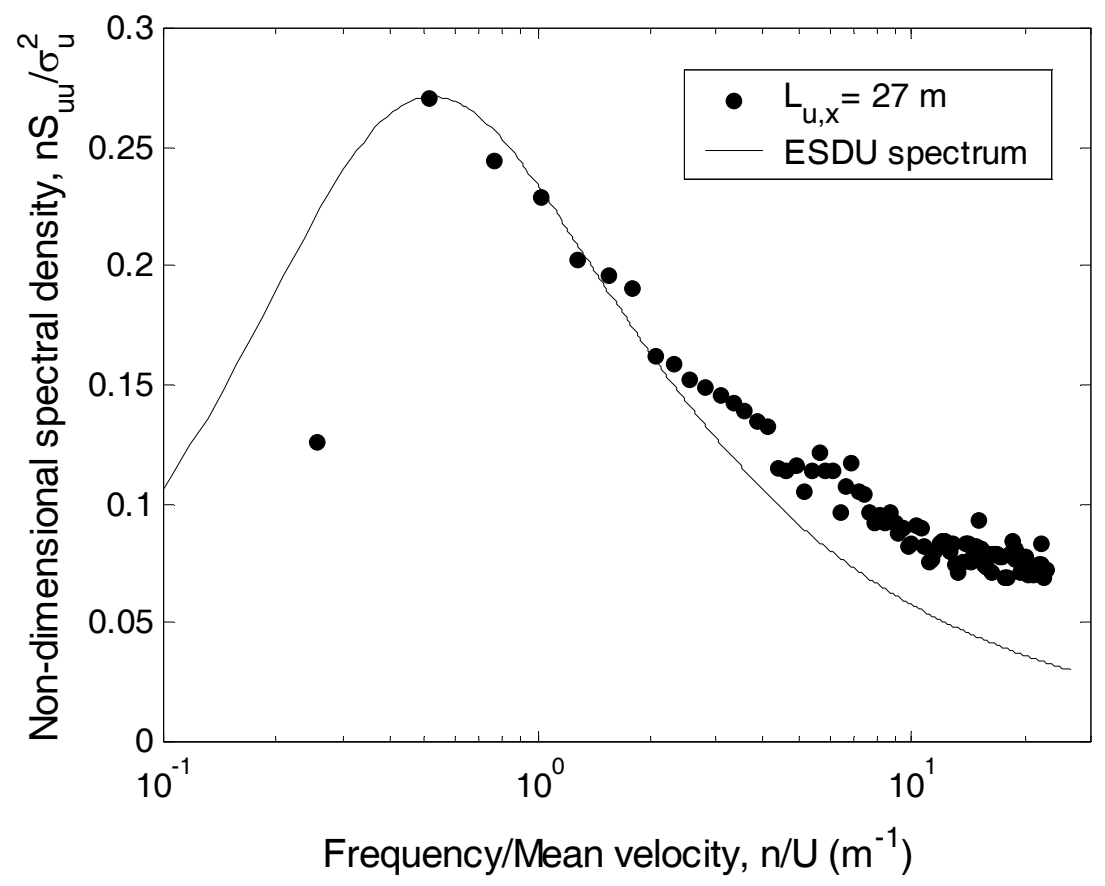

Fig. 4. Turbulence spectrum measured in wind tunnel. 
Lam \& Zhao, Fig. 5
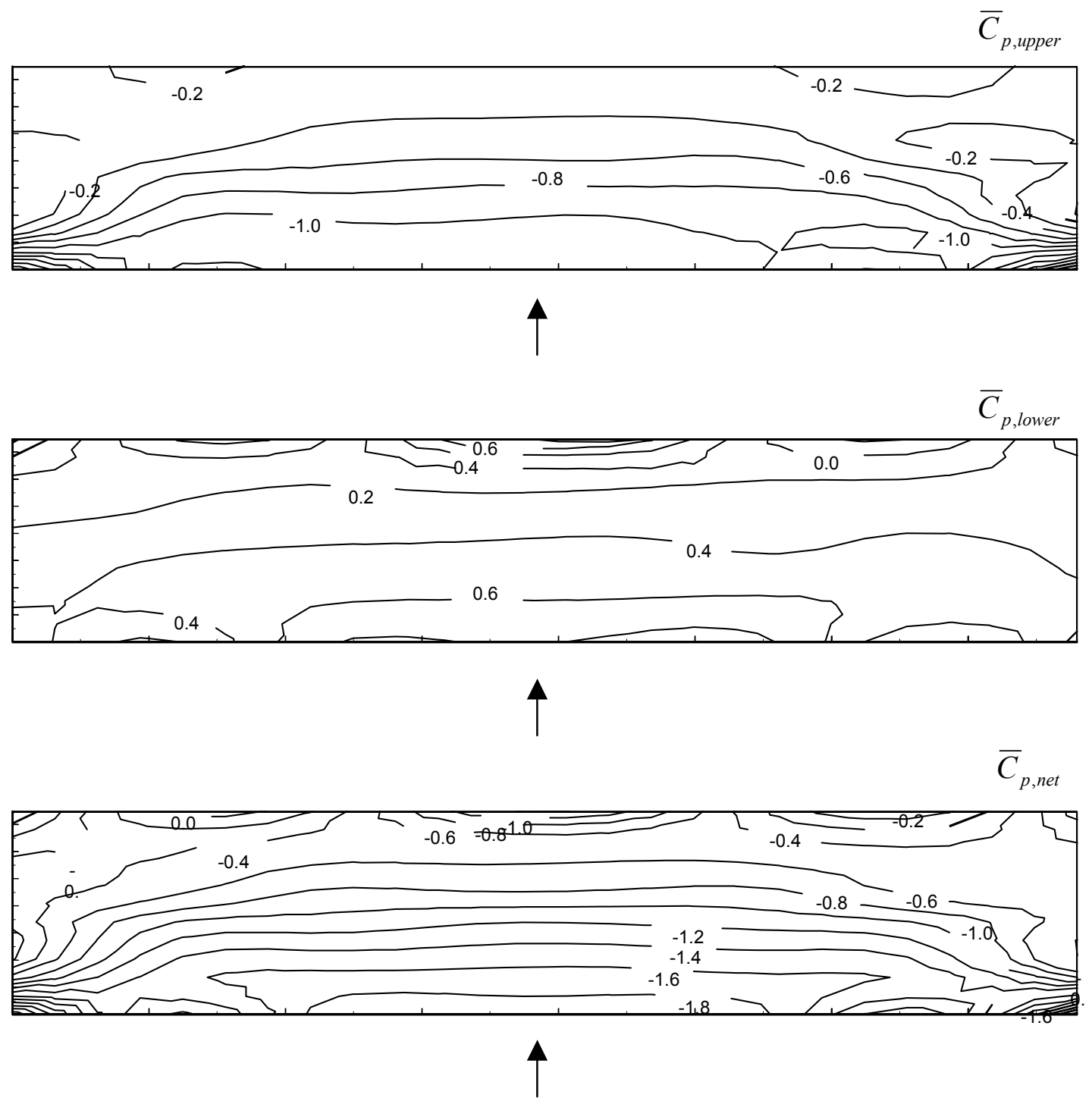

(a) $\alpha=0^{\circ}$

Fig. 5. Contours of mean pressure coefficients on upper roof surface, lower roof surface, and net roof pressure. $\alpha$ : (a) $0^{\circ}$; (b) $20^{\circ}$; (c) $30^{\circ}$; (d) $60^{\circ}$; (e) $90^{\circ}$; (f) $120^{\circ}$; (g) $150^{\circ}$; (h) $180^{\circ}$. 

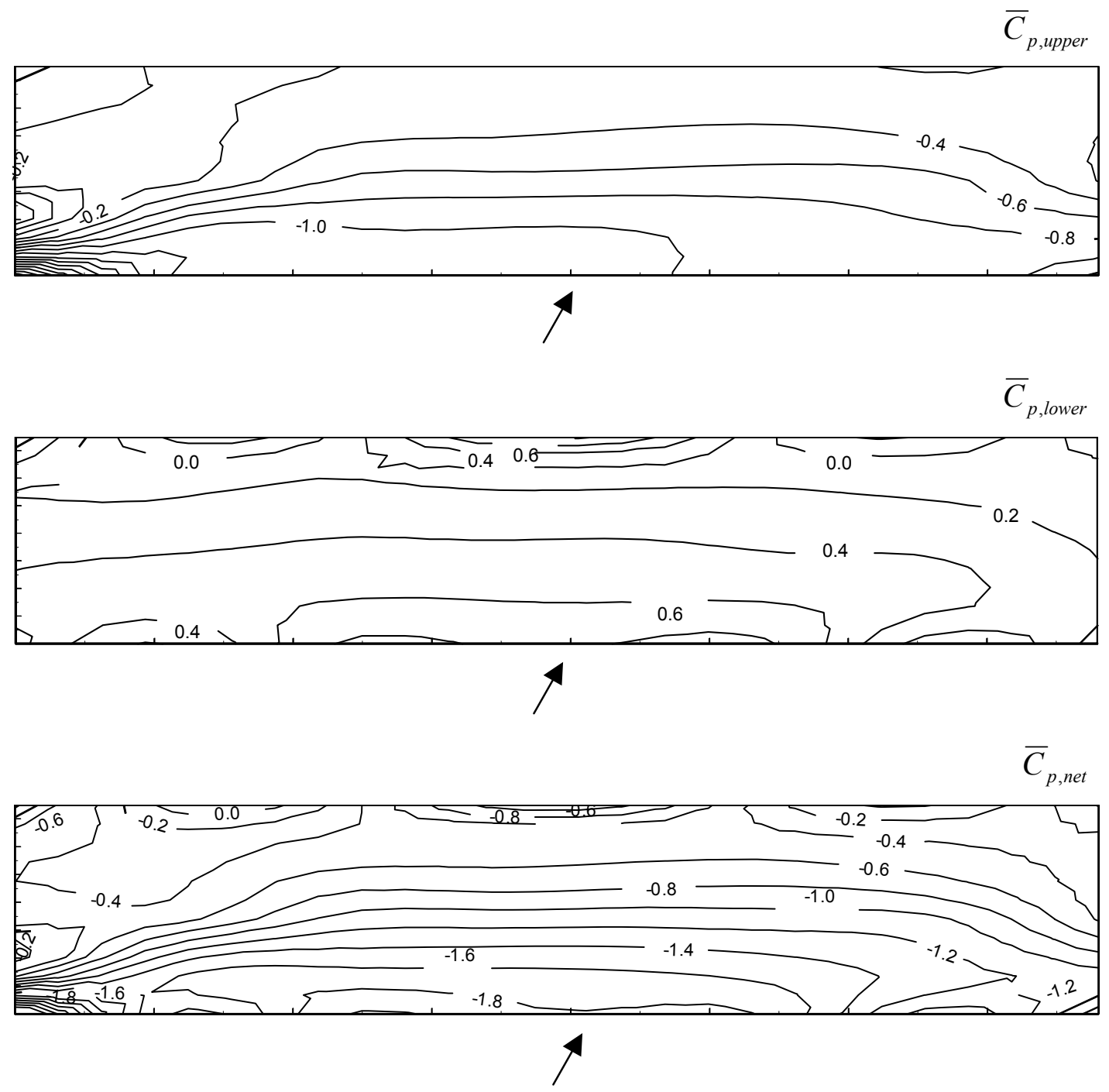

(b) $\alpha=20^{\circ}$

Fig. 5. Contours of mean pressure coefficients on upper roof surface, lower roof surface, and net roof pressure. $\alpha$ : (a) $0^{\circ}$; (b) $20^{\circ}$; (c) $30^{\circ}$; (d) $60^{\circ}$; (e) $90^{\circ}$; (f) $120^{\circ}$; (g) $150^{\circ}$; (h) $180^{\circ}$. 
Lam \& Zhao, Fig. 5
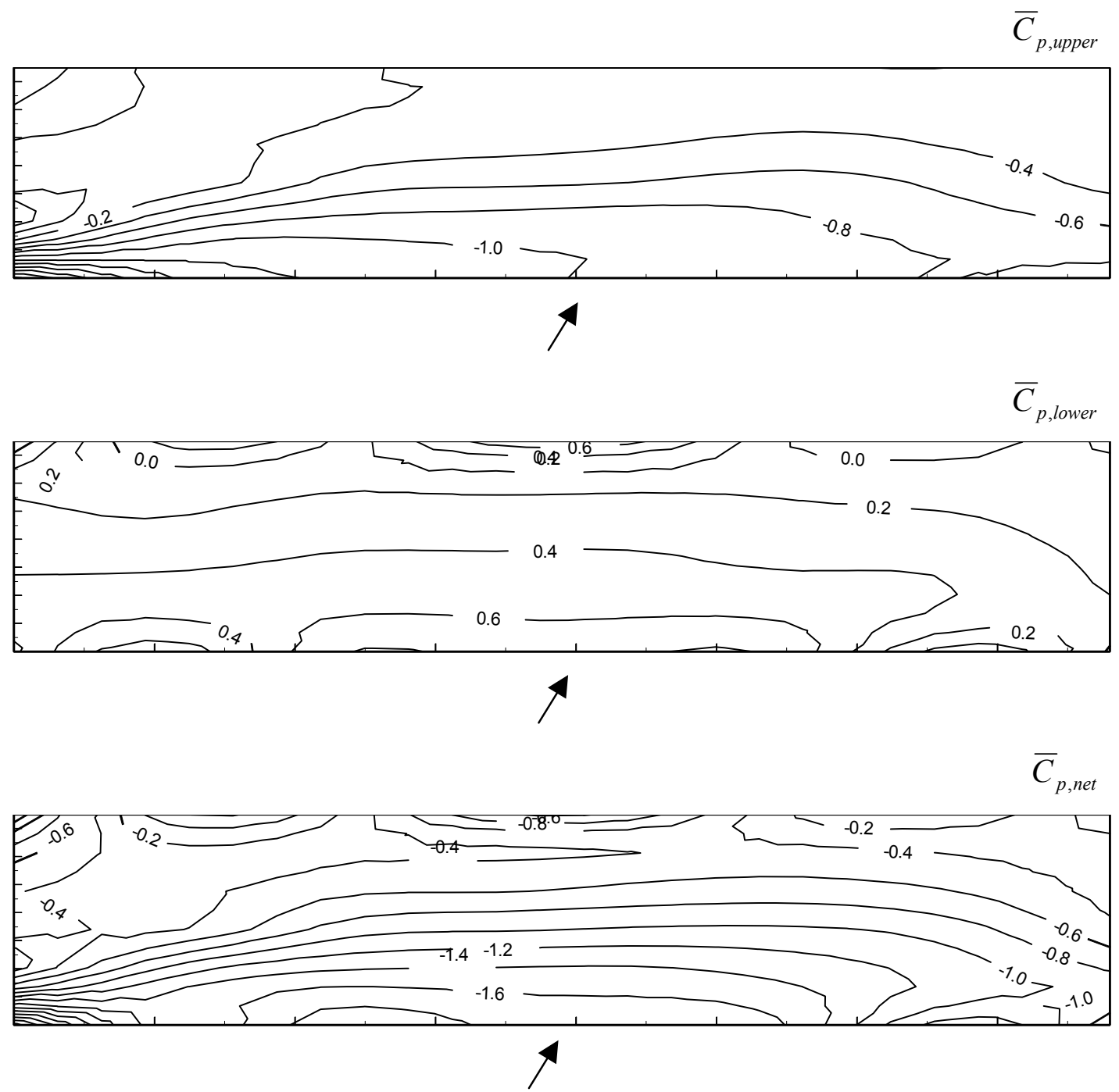

(c) $\alpha=30^{\circ}$

Fig. 5. Contours of mean pressure coefficients on upper roof surface, lower roof surface, and net roof pressure. $\alpha$ : (a) $0^{\circ}$; (b) $20^{\circ}$; (c) $30^{\circ}$; (d) $60^{\circ}$; (e) $90^{\circ}$; (f) $120^{\circ}$; (g) $150^{\circ}$; (h) $180^{\circ}$. 
Lam \& Zhao, Fig. 5
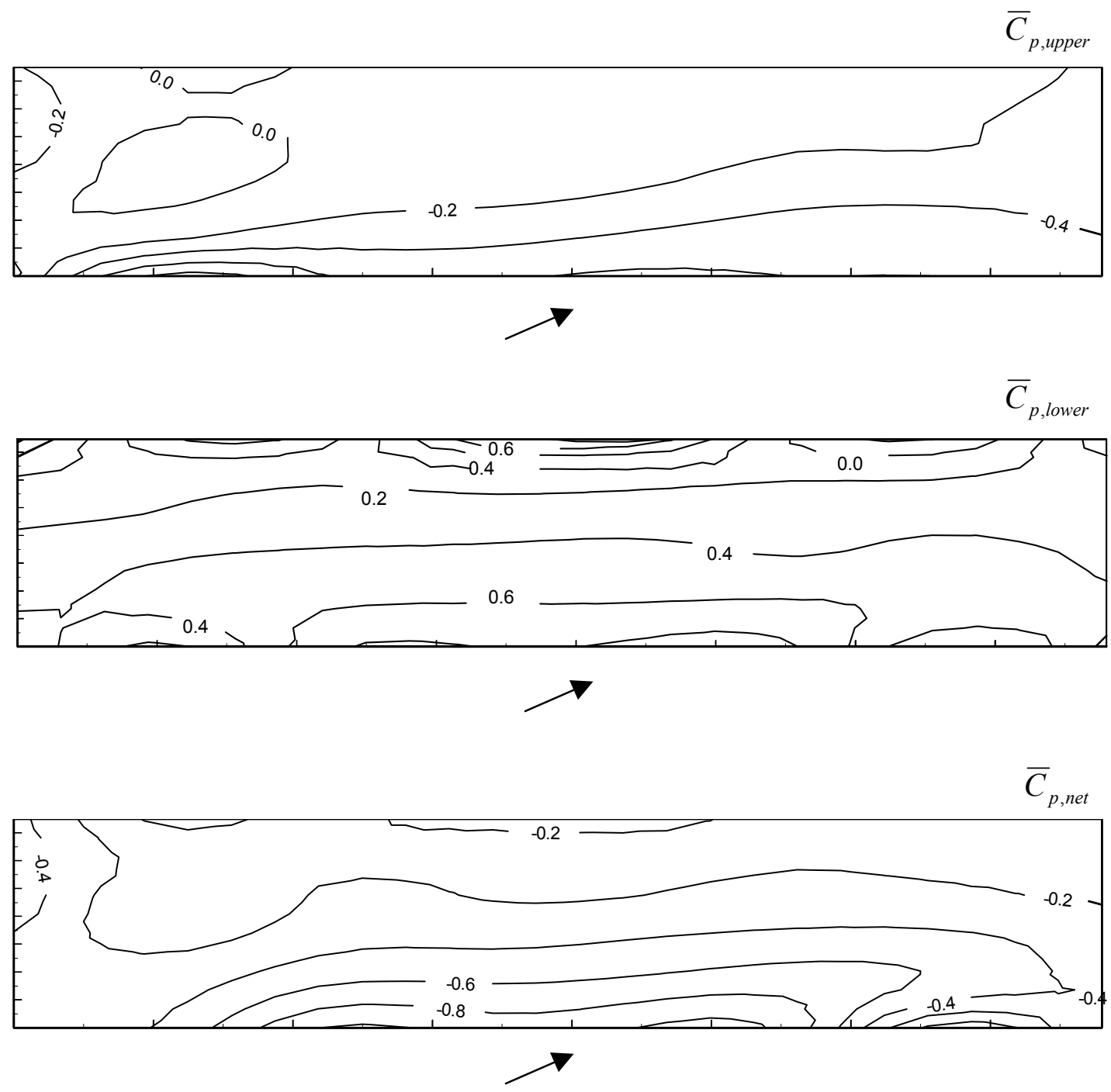

(d) $\alpha=60^{\circ}$

Fig. 5. Contours of mean pressure coefficients on upper roof surface, lower roof surface, and net roof pressure. $\alpha$ : (a) $0^{\circ}$; (b) $20^{\circ}$; (c) $30^{\circ}$; (d) $60^{\circ}$; (e) $90^{\circ}$; (f) $120^{\circ}$; (g) $150^{\circ}$; (h) $180^{\circ}$. 

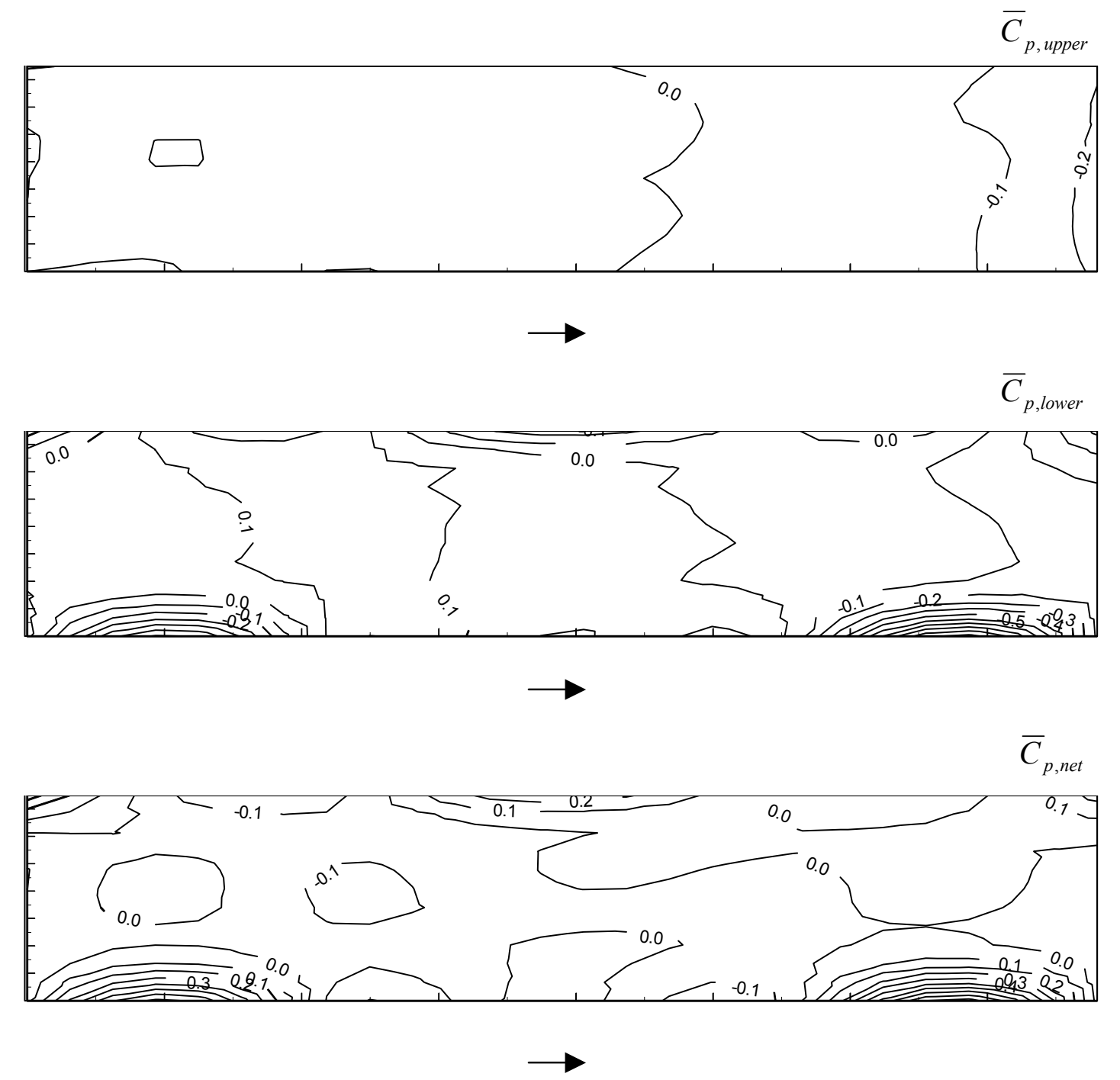

(e) $\alpha=90^{\circ}$

Fig. 5. Contours of mean pressure coefficients on upper roof surface, lower roof surface, and net roof pressure. $\alpha$ : (a) $0^{\circ}$; (b) $20^{\circ}$; (c) $30^{\circ}$; (d) $60^{\circ}$; (e) $90^{\circ}$; (f) $120^{\circ}$; (g) $150^{\circ}$; (h) $180^{\circ}$. 

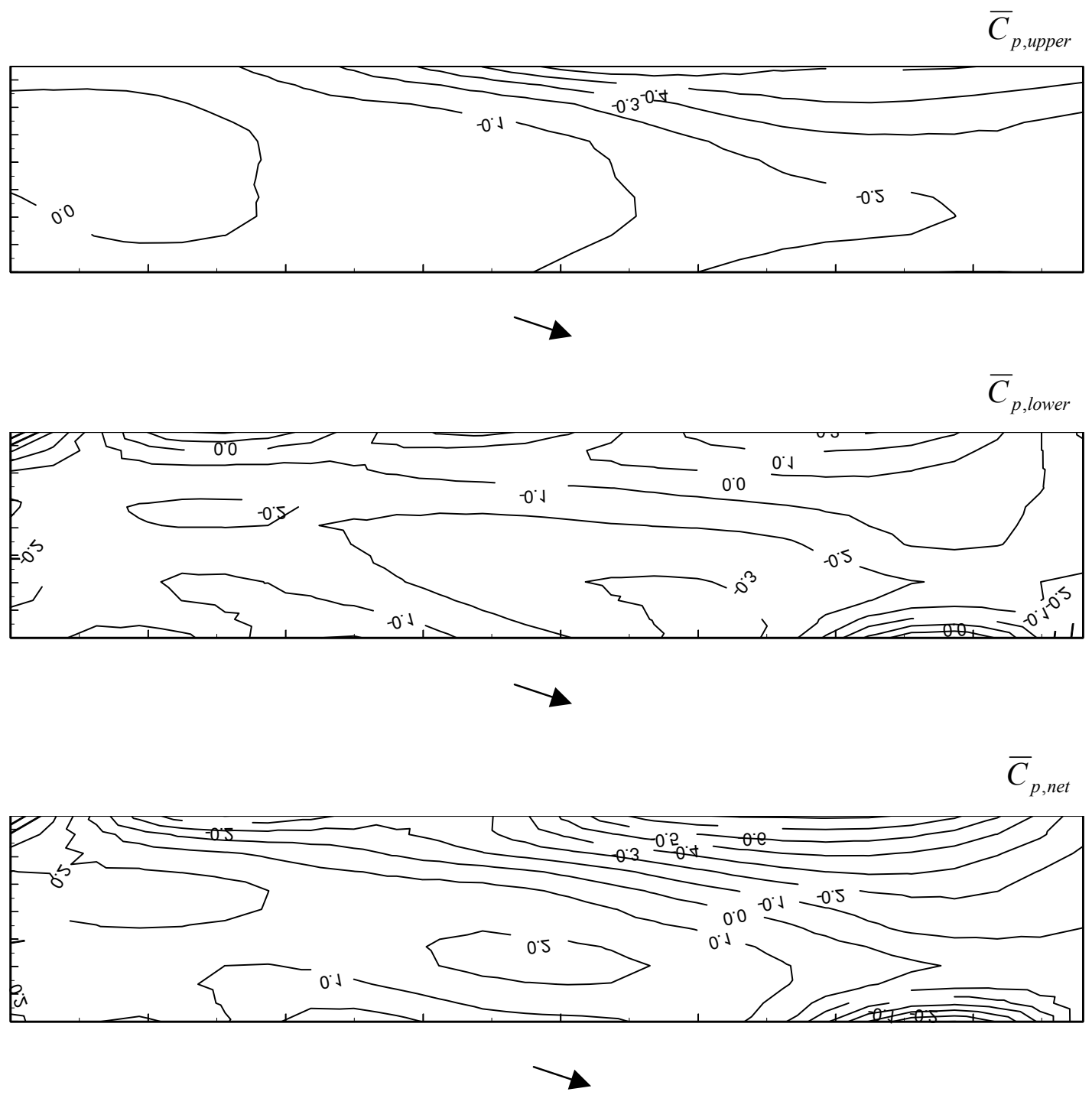

(f) $\alpha=120^{\circ}$

Fig. 5. Contours of mean pressure coefficients on upper roof surface, lower roof surface, and net roof pressure. $\alpha$ : (a) $0^{\circ}$; (b) $20^{\circ}$; (c) $30^{\circ}$; (d) $60^{\circ}$; (e) $90^{\circ}$; (f) $120^{\circ}$; (g) $150^{\circ}$; (h) $180^{\circ}$. 
Lam \& Zhao, Fig. 5
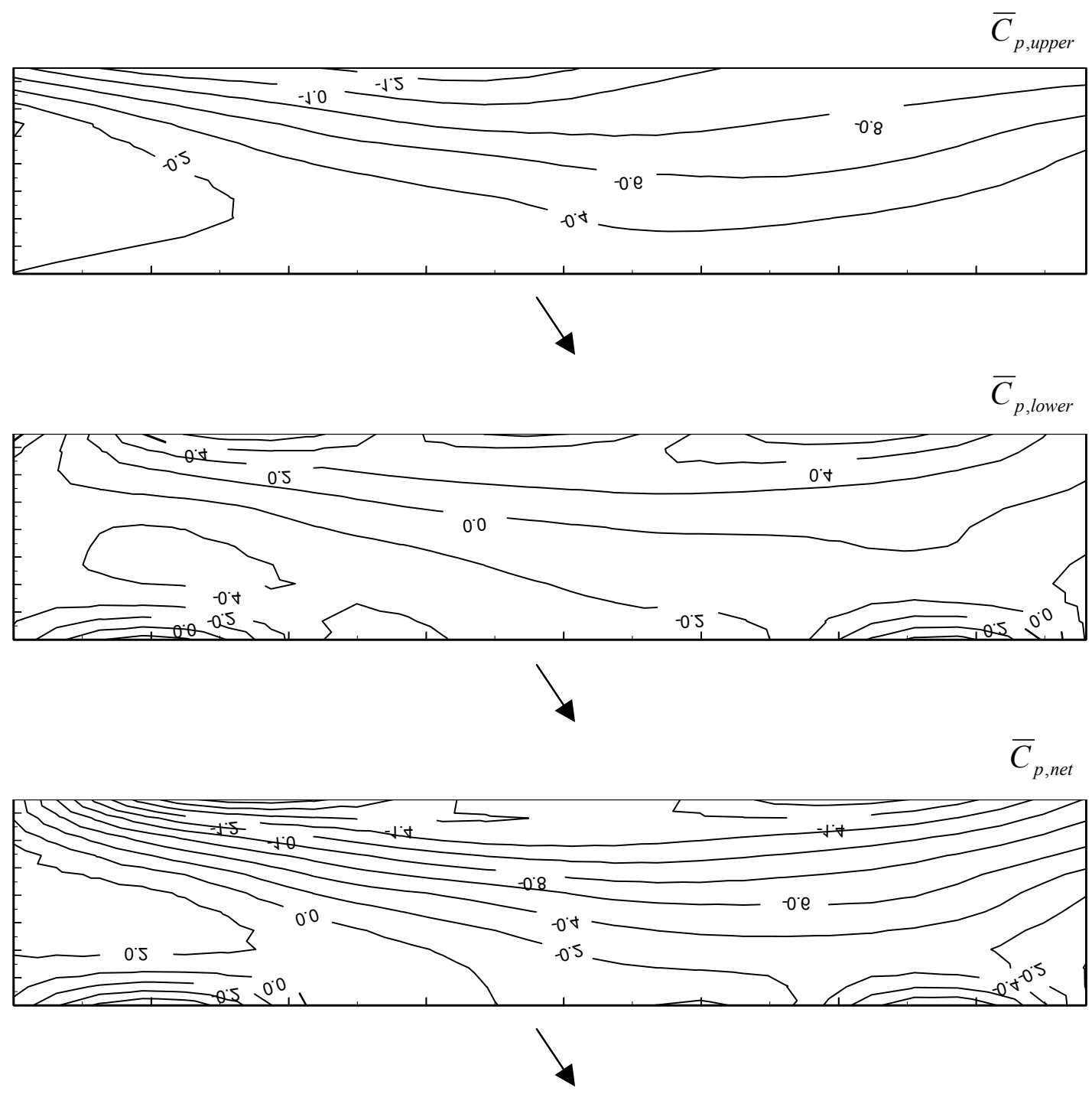

(g) $\alpha=150^{\circ}$

Fig. 5. Contours of mean pressure coefficients on upper roof surface, lower roof surface, and net roof pressure. $\alpha$ : (a) $0^{\circ}$; (b) $20^{\circ}$; (c) $30^{\circ}$; (d) $60^{\circ}$; (e) $90^{\circ}$; (f) $120^{\circ}$; (g) $150^{\circ}$; (h) $180^{\circ}$. 
Lam \& Zhao, Fig. 5
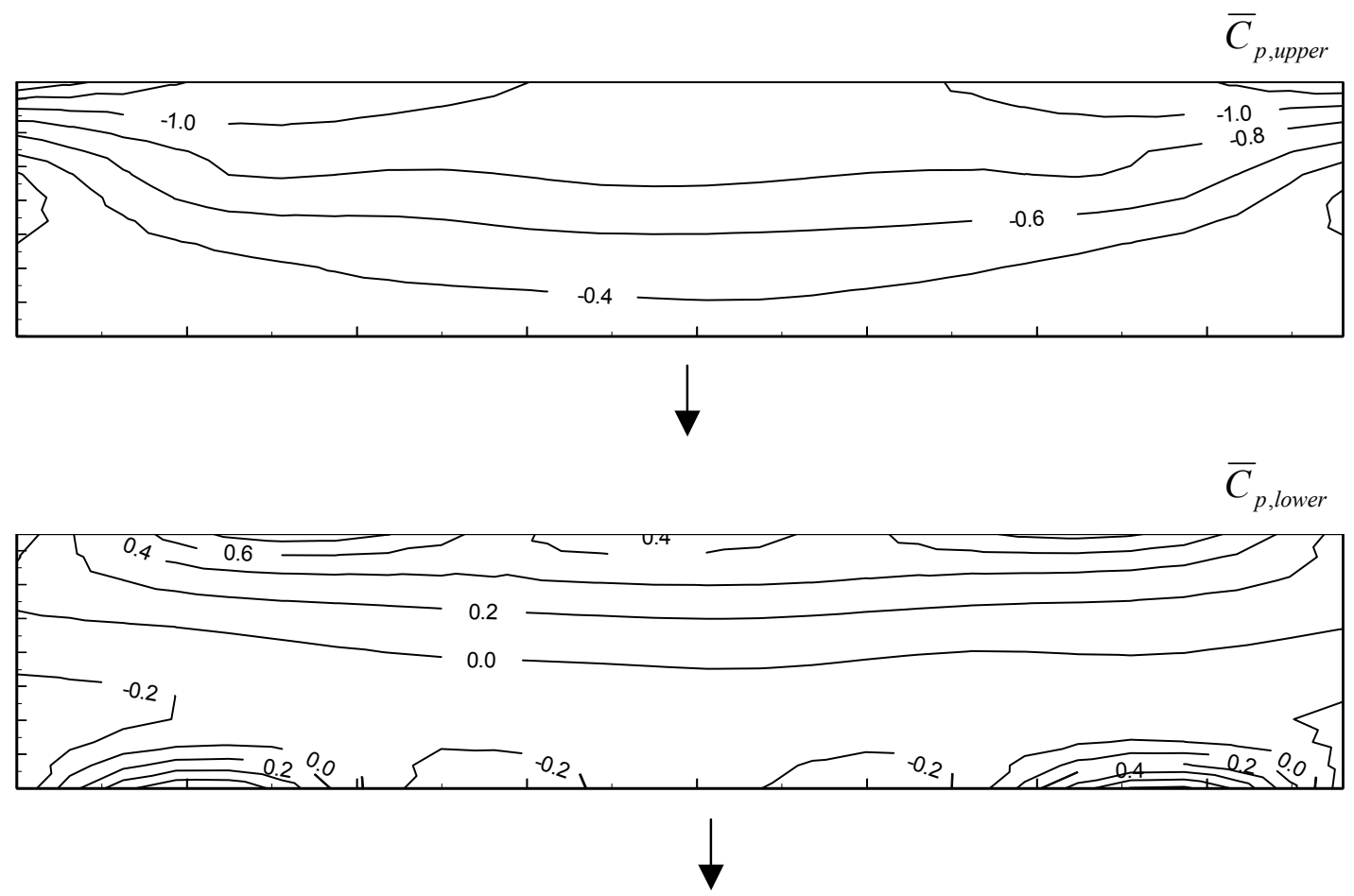

$\bar{C}_{p, n e t}$

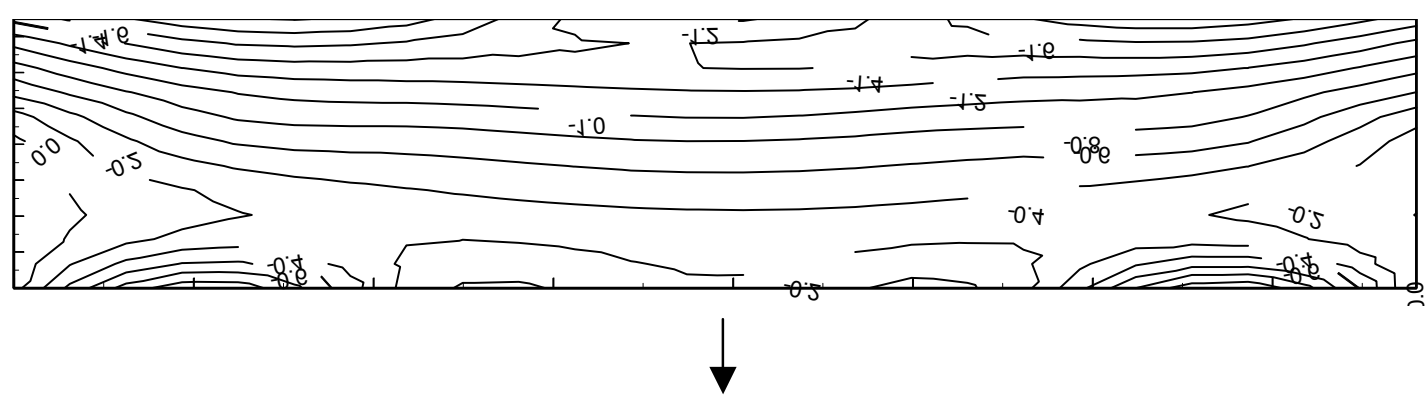

(h) $\alpha=180^{\circ}$

Fig. 5. Contours of mean pressure coefficients on upper roof surface, lower roof surface, and net roof pressure. $\alpha$ : (a) $0^{\circ}$; (b) $20^{\circ}$; (c) $30^{\circ}$; (d) $60^{\circ}$; (e) $90^{\circ}$; (f) $120^{\circ}$; (g) $150^{\circ}$; (h) $180^{\circ}$. 

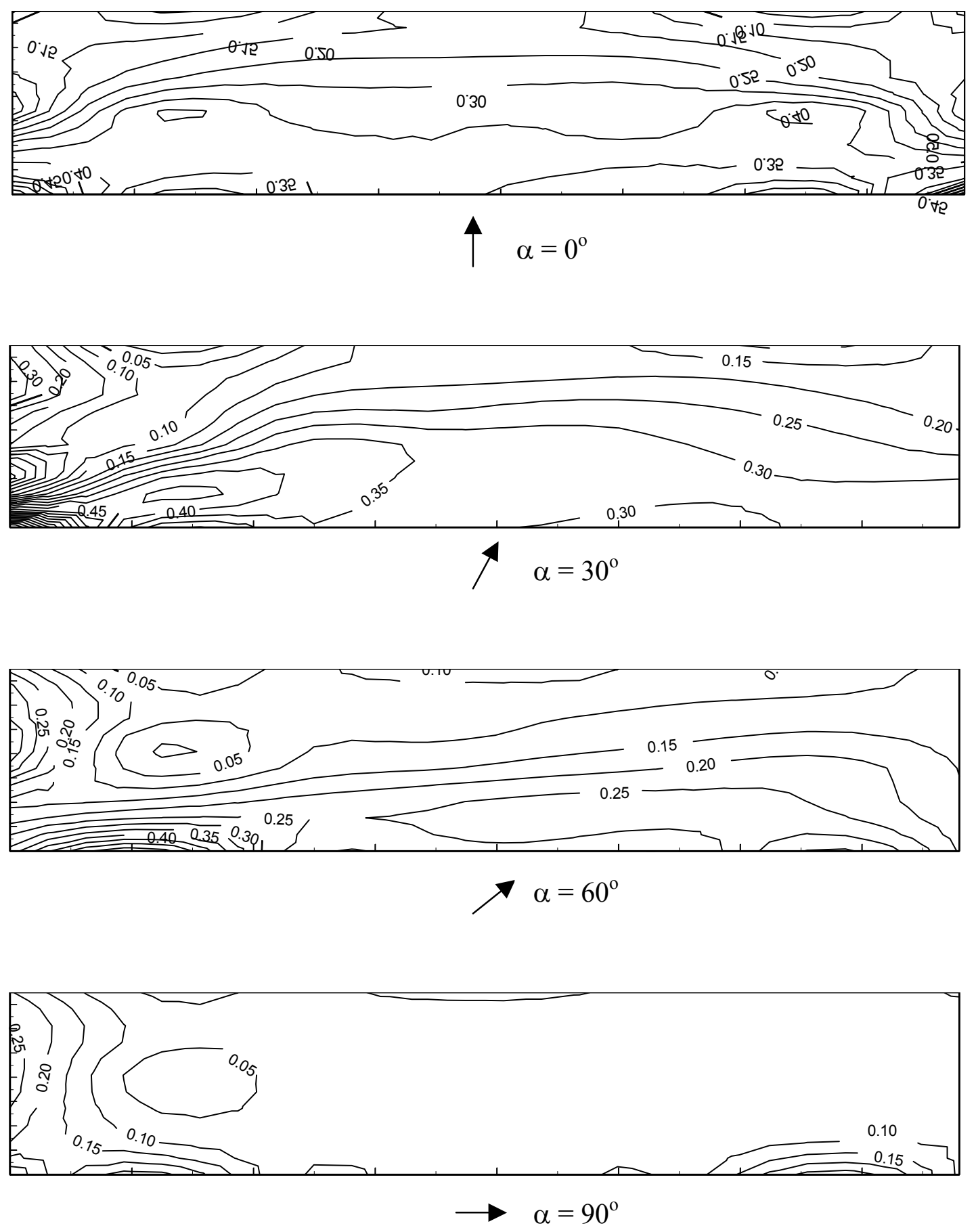

Fig. 6. Contours of pressure coefficients of standard deviation of net roof pressure fluctuations. 
Lam \& Zhao, Fig. 6

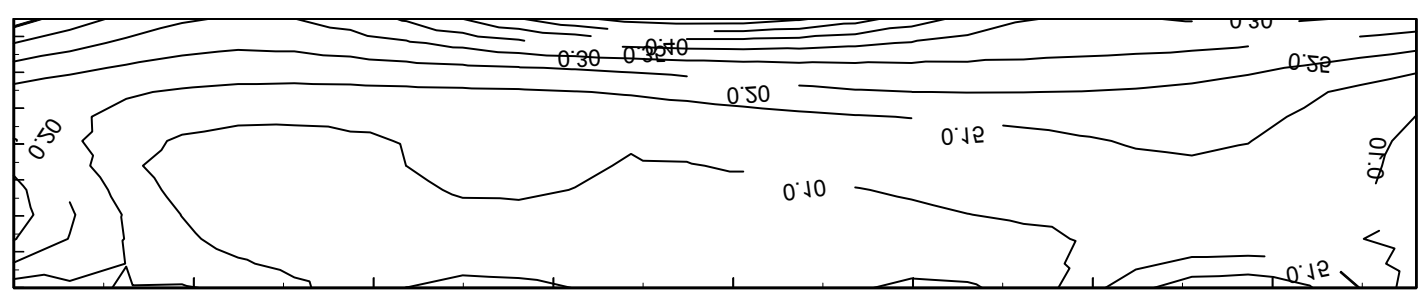

$\triangle \alpha=120^{\circ}$
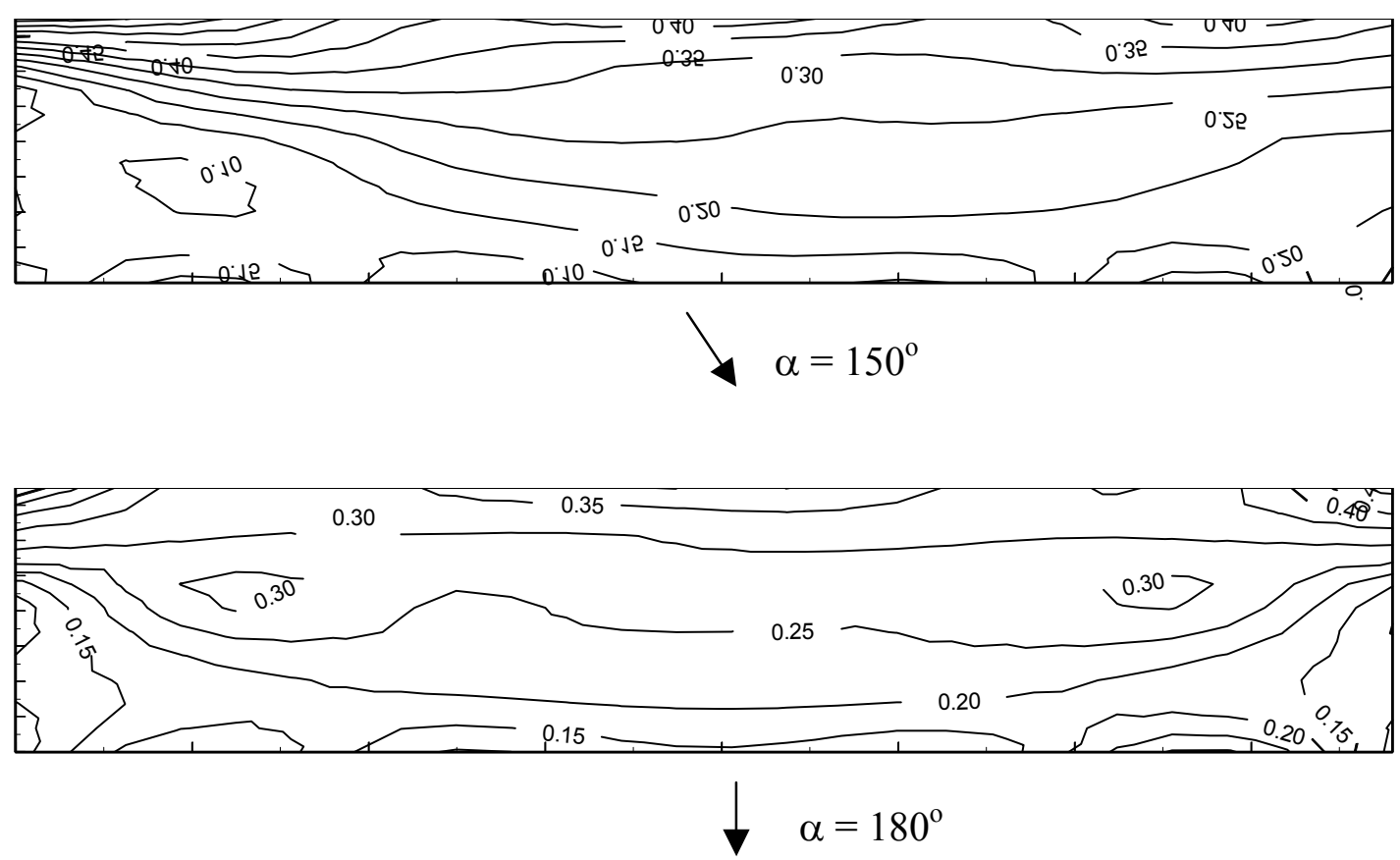

Fig. 6. Contours of pressure coefficients of standard deviation of net roof pressure fluctuations. 

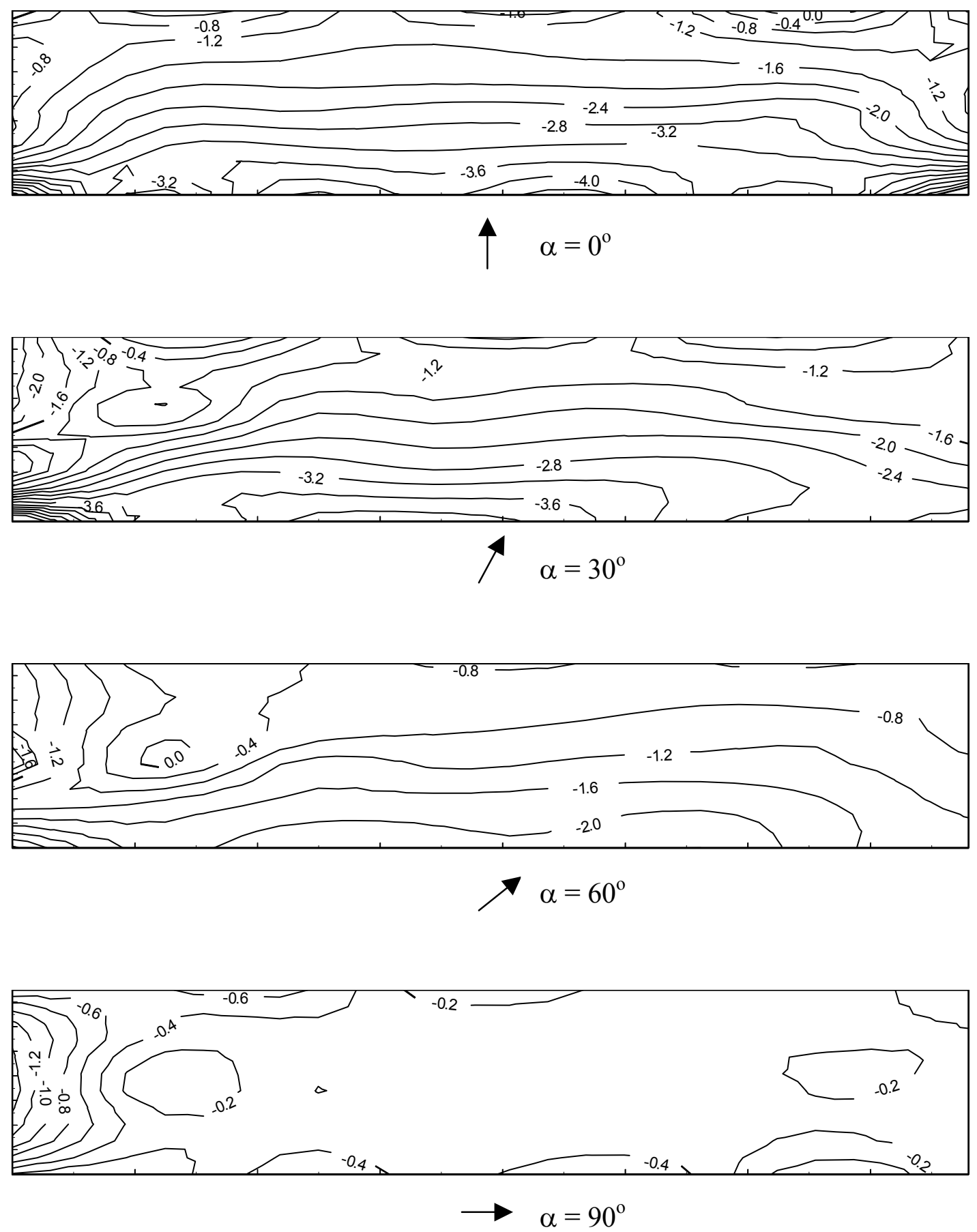

(a) peak minimum pressure coefficient

Fig. 7. Contours of peak net roof pressure coefficients. (a) peak minimum pressure coefficient; (b) peak maximum pressure coefficient. 


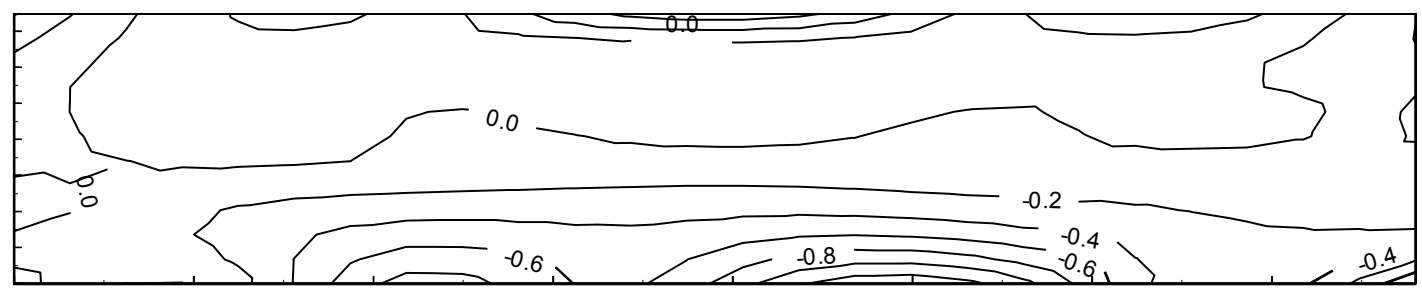

$\uparrow \alpha=0^{\circ}$
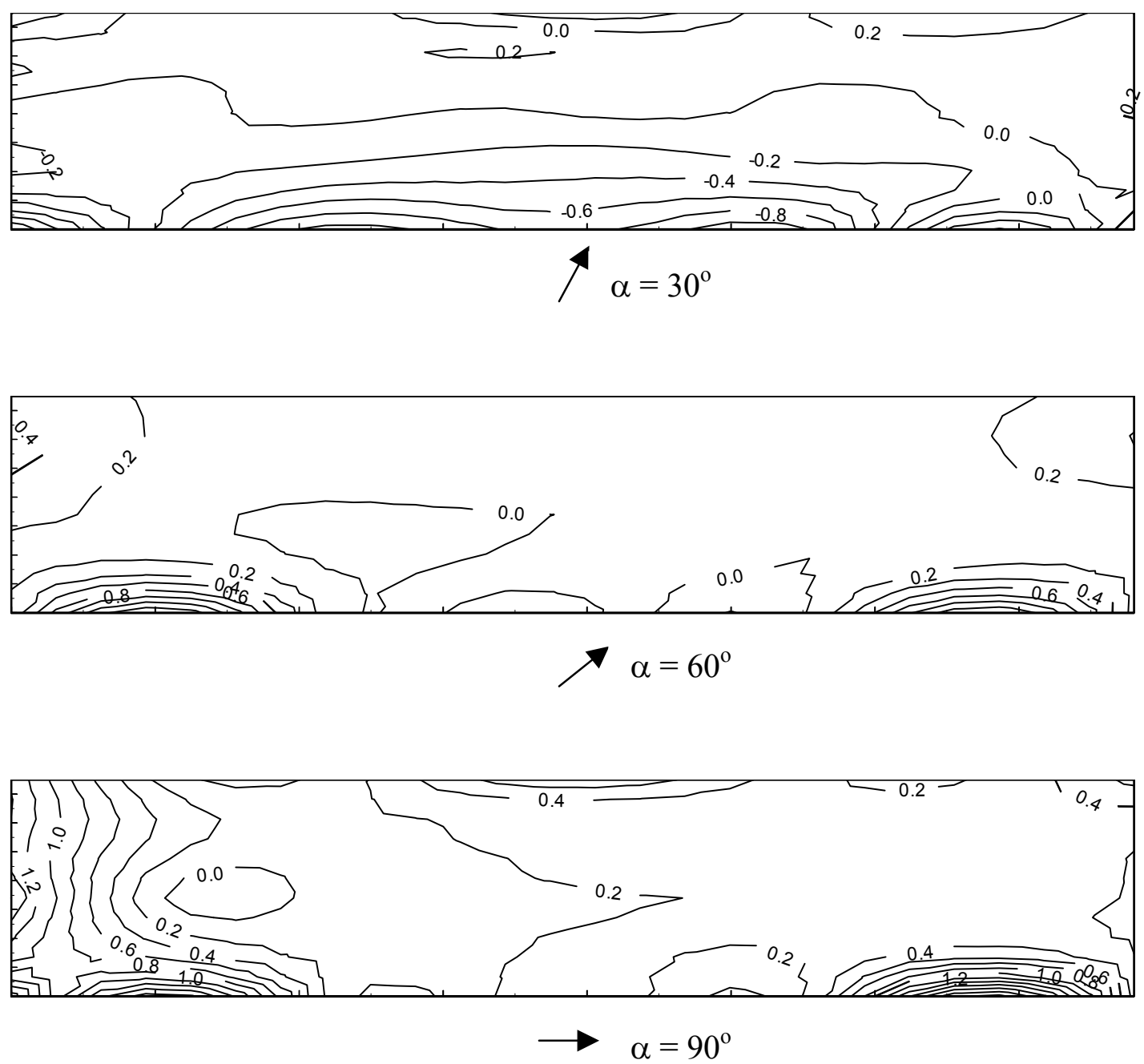

(b) peak maximum pressure coefficient

Fig. 7. Contours of peak net roof pressure coefficients. (a) peak minimum pressure coefficient; (b) peak maximum pressure coefficient. 


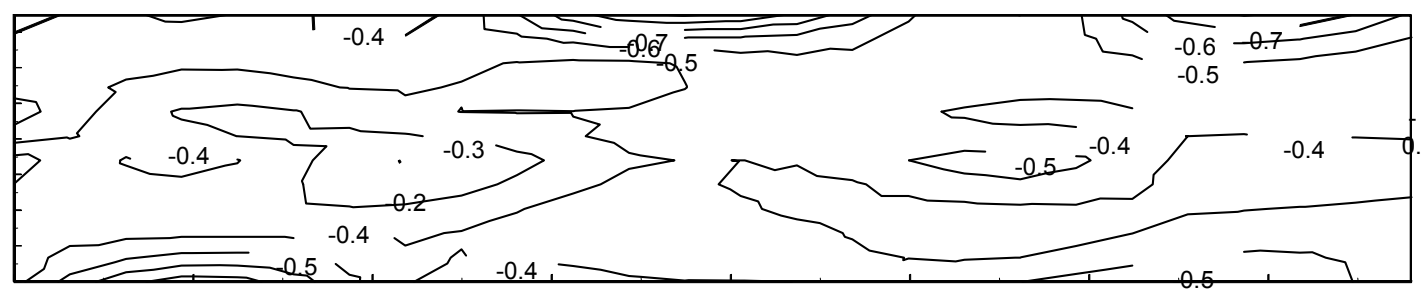

$\hat{\uparrow} \alpha=0^{\circ}$
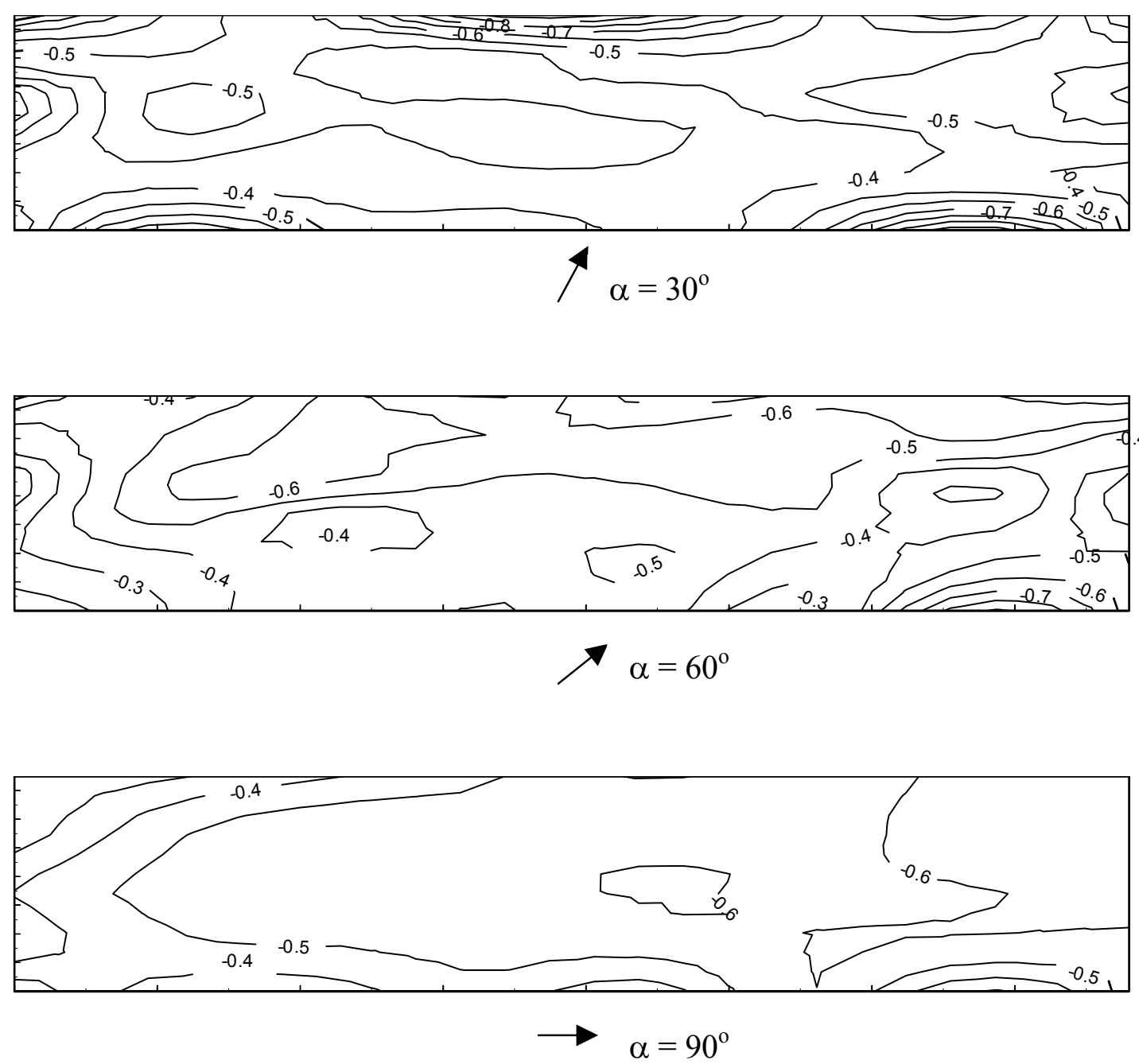

(a) peak minimum pressure coefficient

Fig. 8. Amount of overestimation of peak net pressure from worst case combination of peak values from two surfaces. (a) peak minimum pressure coefficient; (b) peak maximum pressure coefficient. 
Lam \& Zhao, Fig. 8

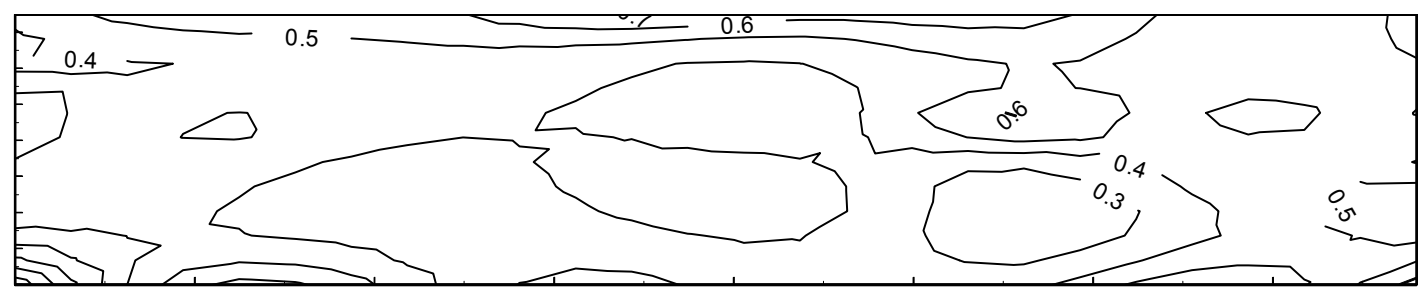

$\uparrow \alpha=0^{\circ}$
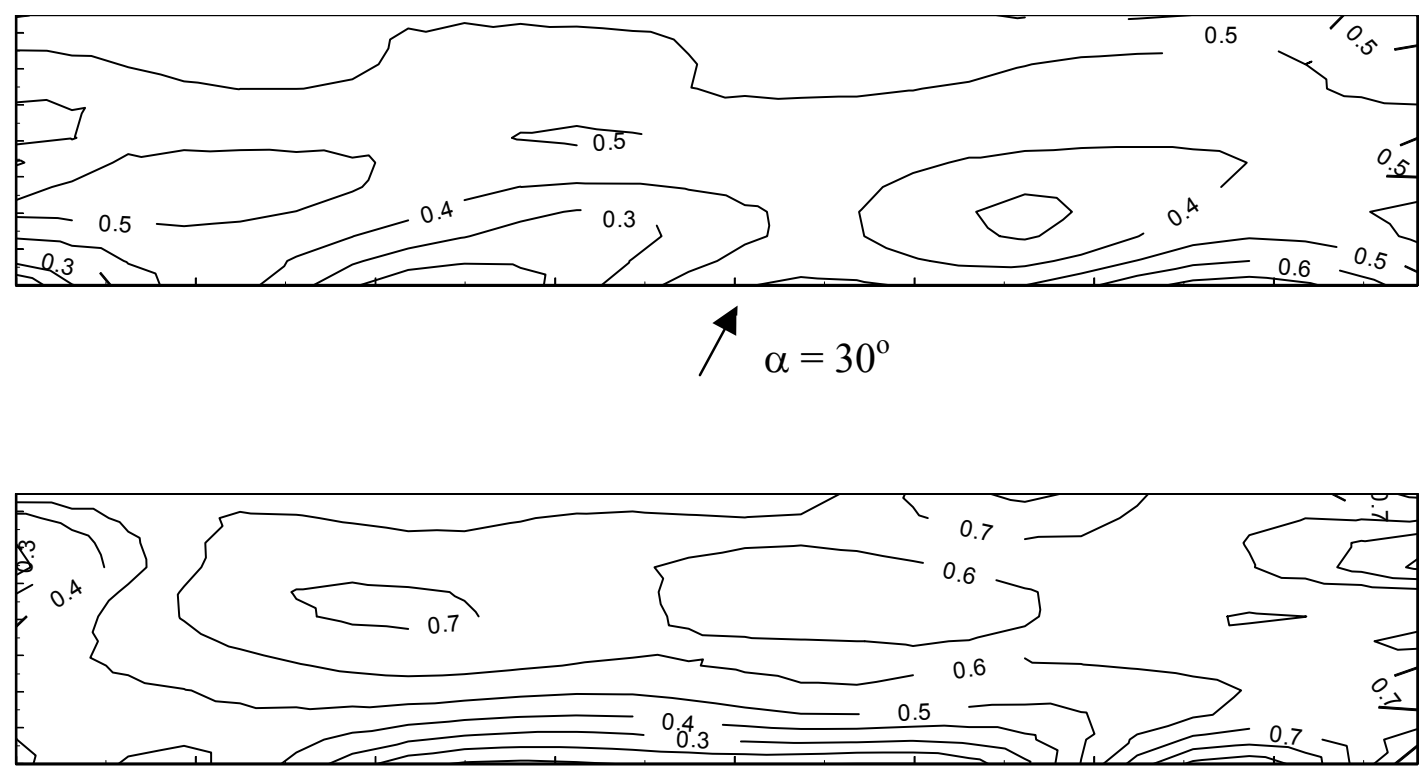

$\alpha=60^{\circ}$

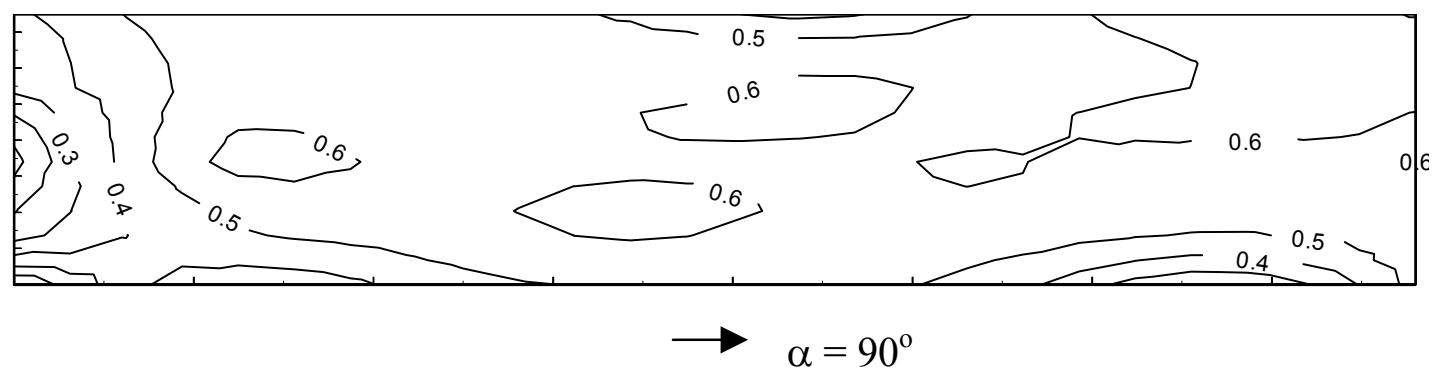

(b) peak maximum pressure coefficient

Fig. 8. Amount of overestimation of peak net pressure from worst case combination of peak values from two surfaces. (a) peak minimum pressure coefficient; (b) peak maximum pressure coefficient. 
Lam \& Zhao, Fig. 9

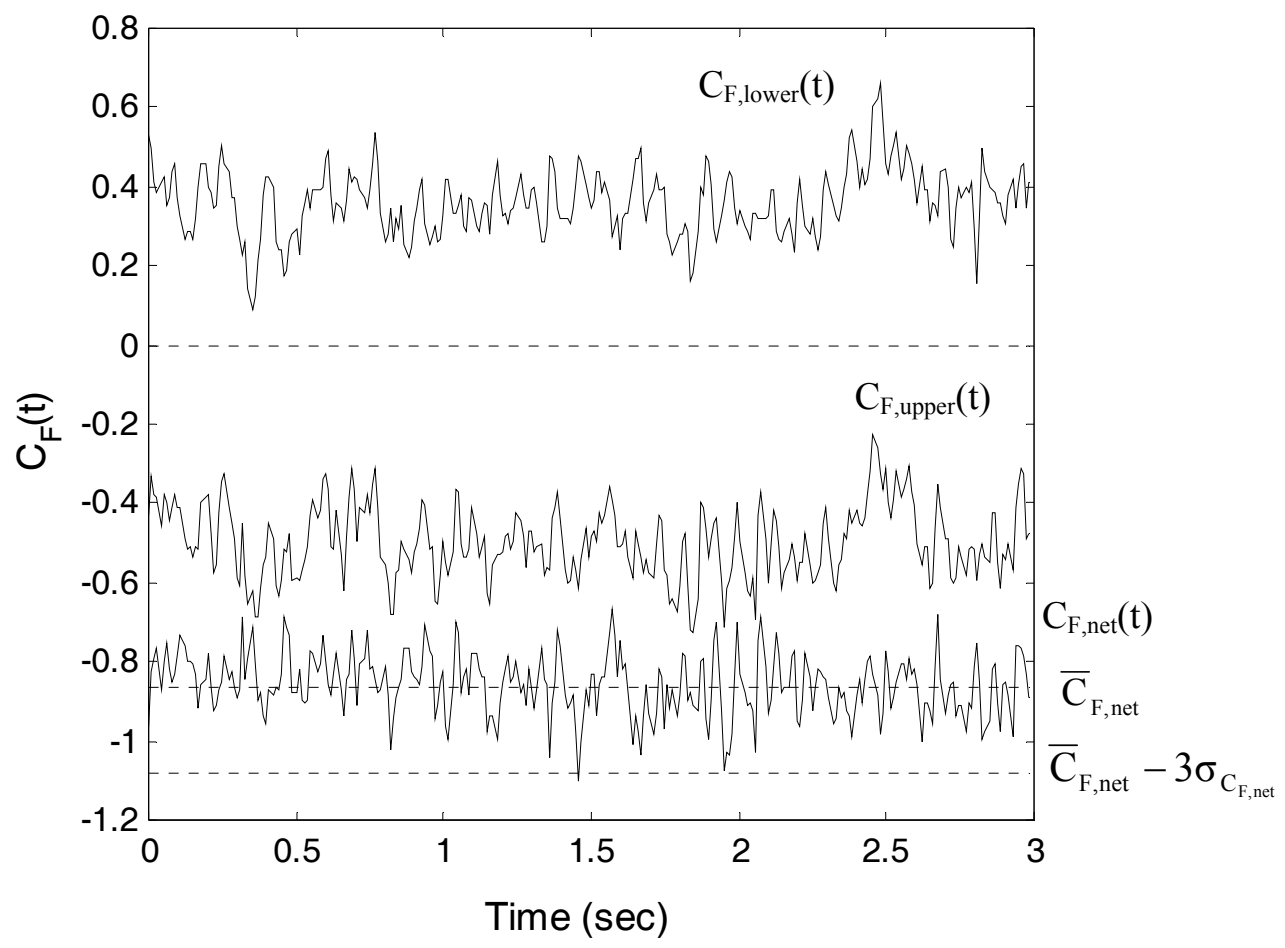

Fig. 9. Time signals of wind force coefficients on different roof surfaces at $\alpha=30^{\circ}$. 
Lam \& Zhao, Fig. 10
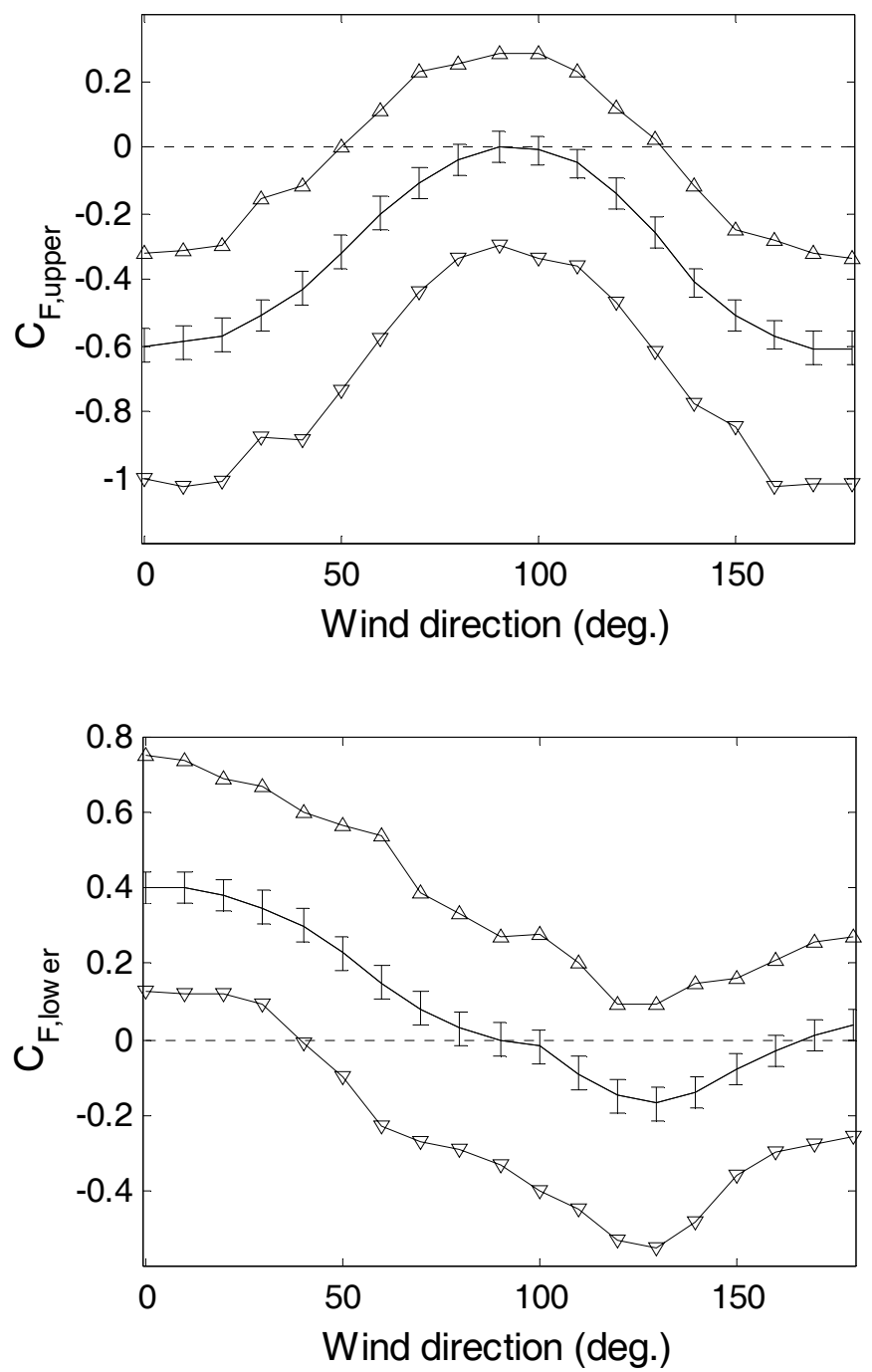

(b)

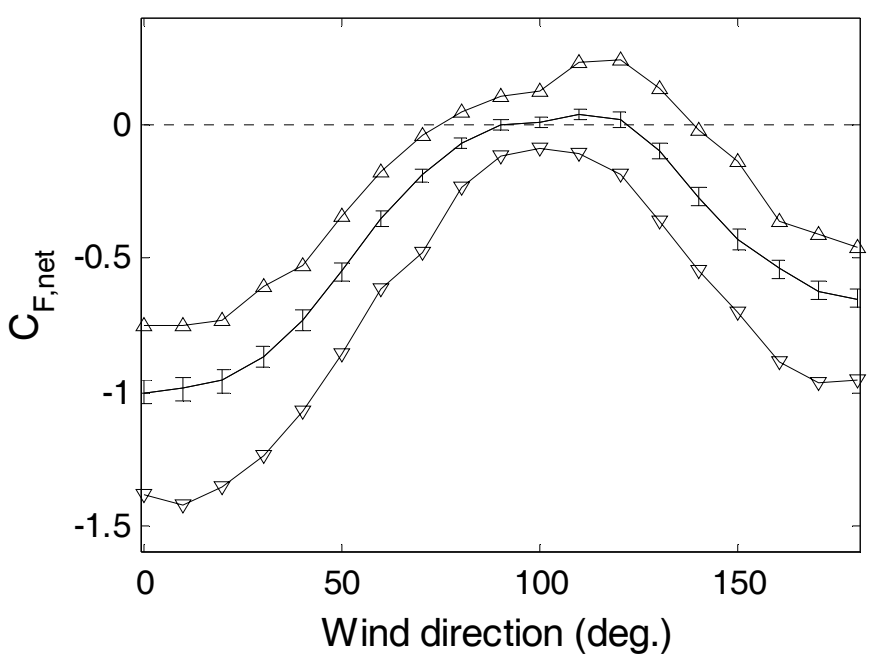

(c) 

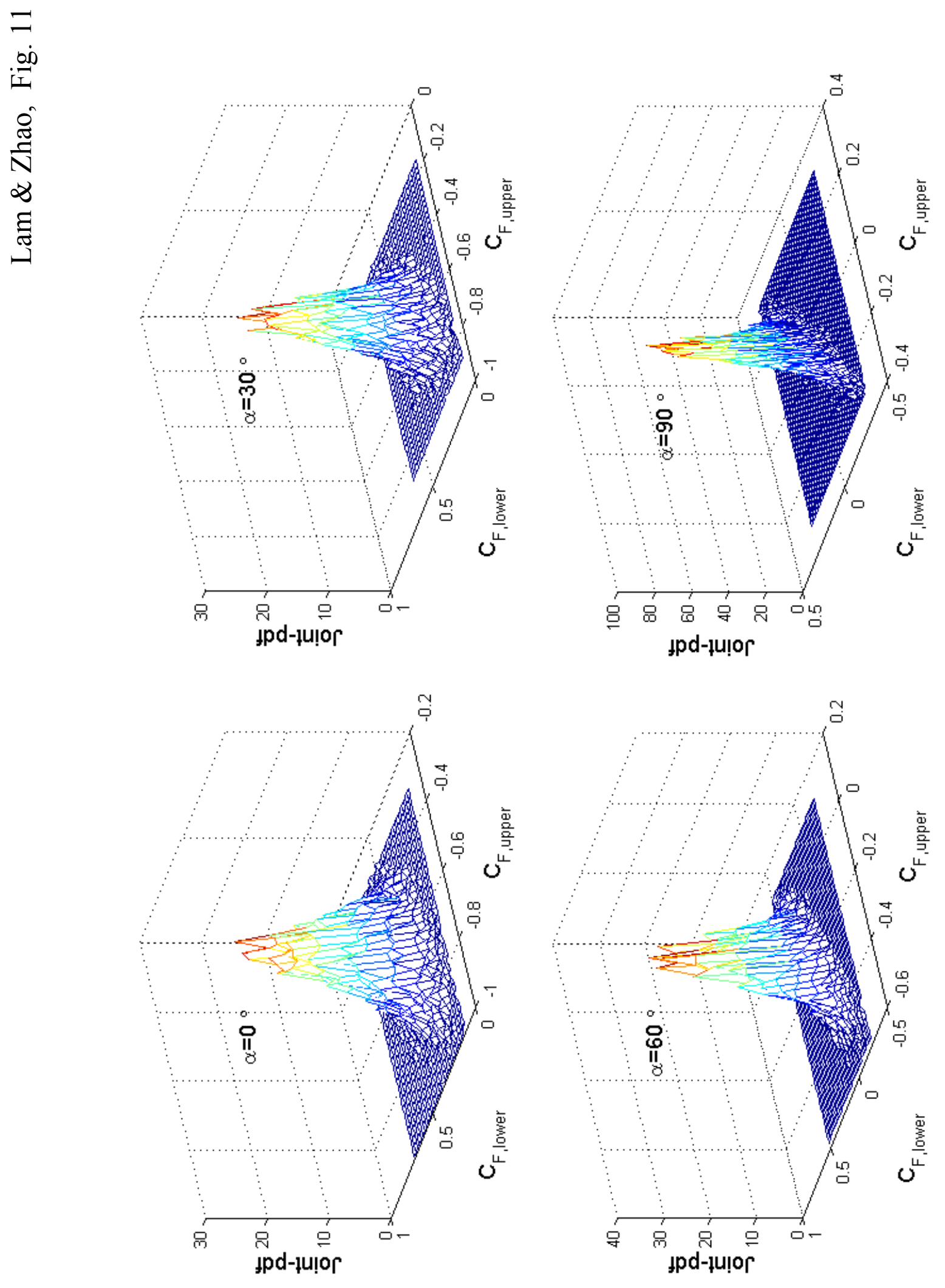
Lam \& Zhao, Fig. 12
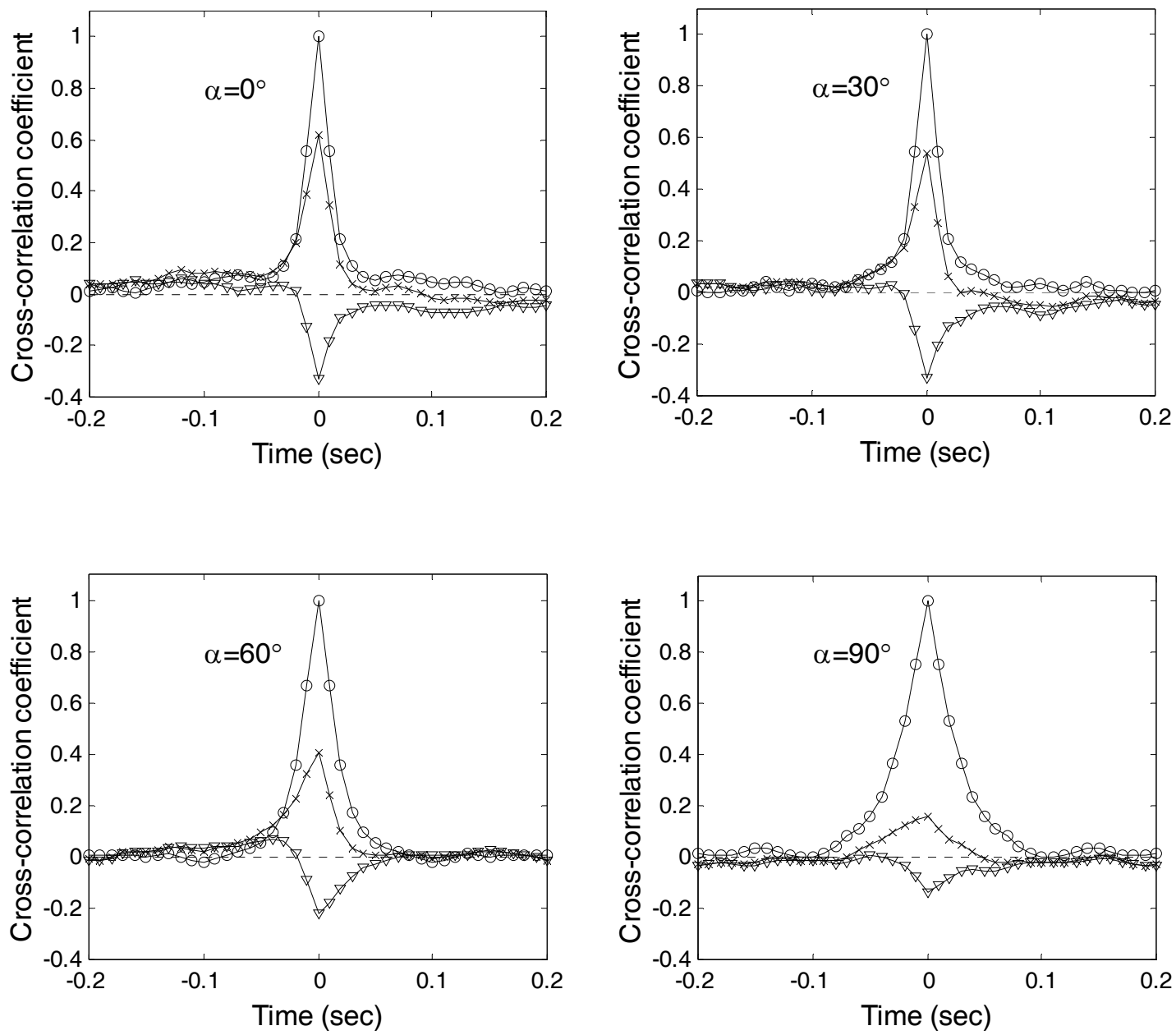

Fig. 12. Cross-correlation coefficients between total net roof force and upper/lower surface forces. Curves: o, auto-correlation of net roof force; $\times$, net roof force with upper surface force; $\nabla$, net roof force with lower surface force. 
Lam \& Zhao, Fig. 13
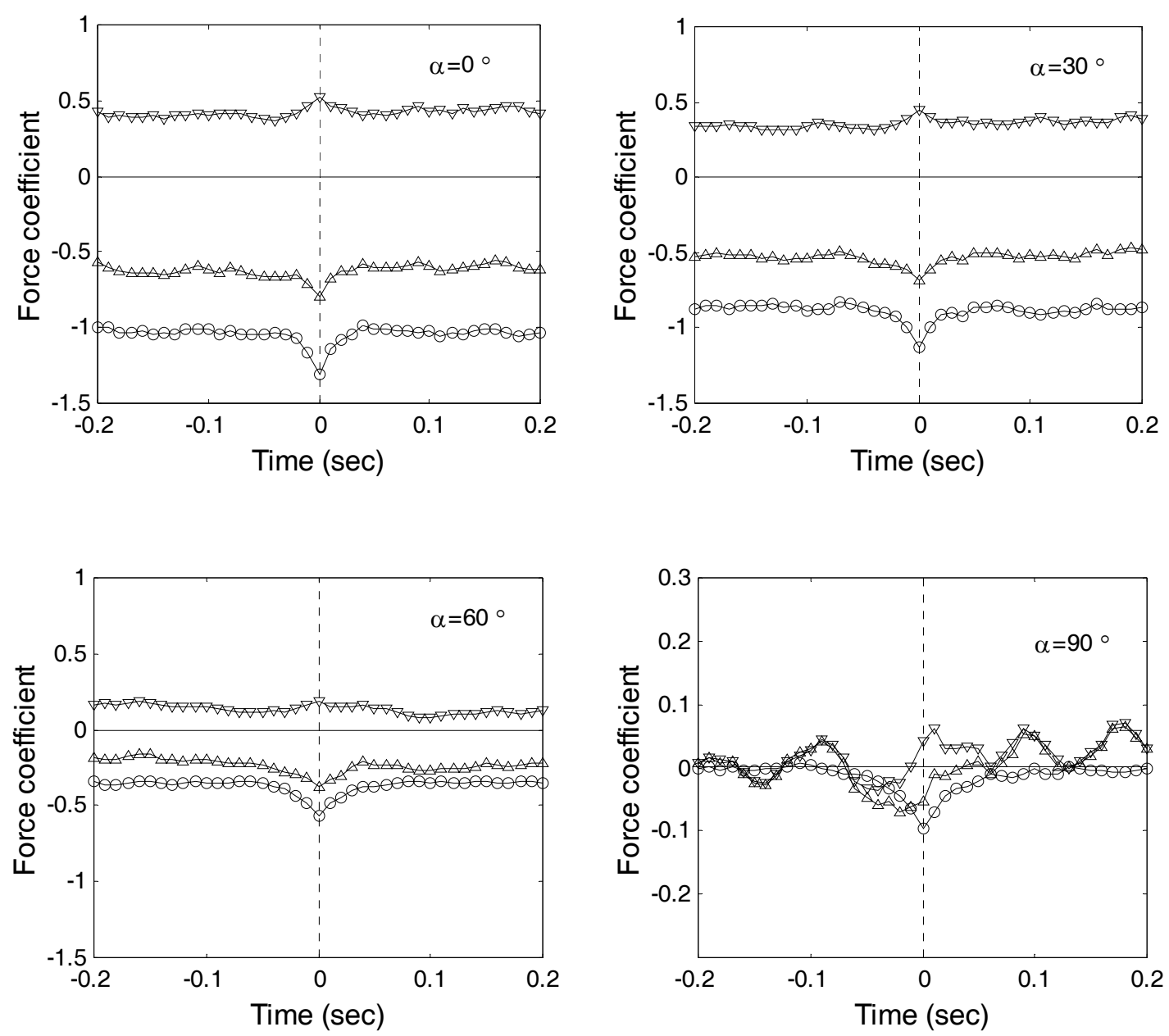

Fig. 13. Roof surface force coefficients conditionally sampled by peak total roof lift.

Curves: o, self-sampled total roof force coefficient;

$\Delta$, conditionally sampled upper roof force coefficient;

$\nabla$, conditionally sampled lower roof force coefficient. 

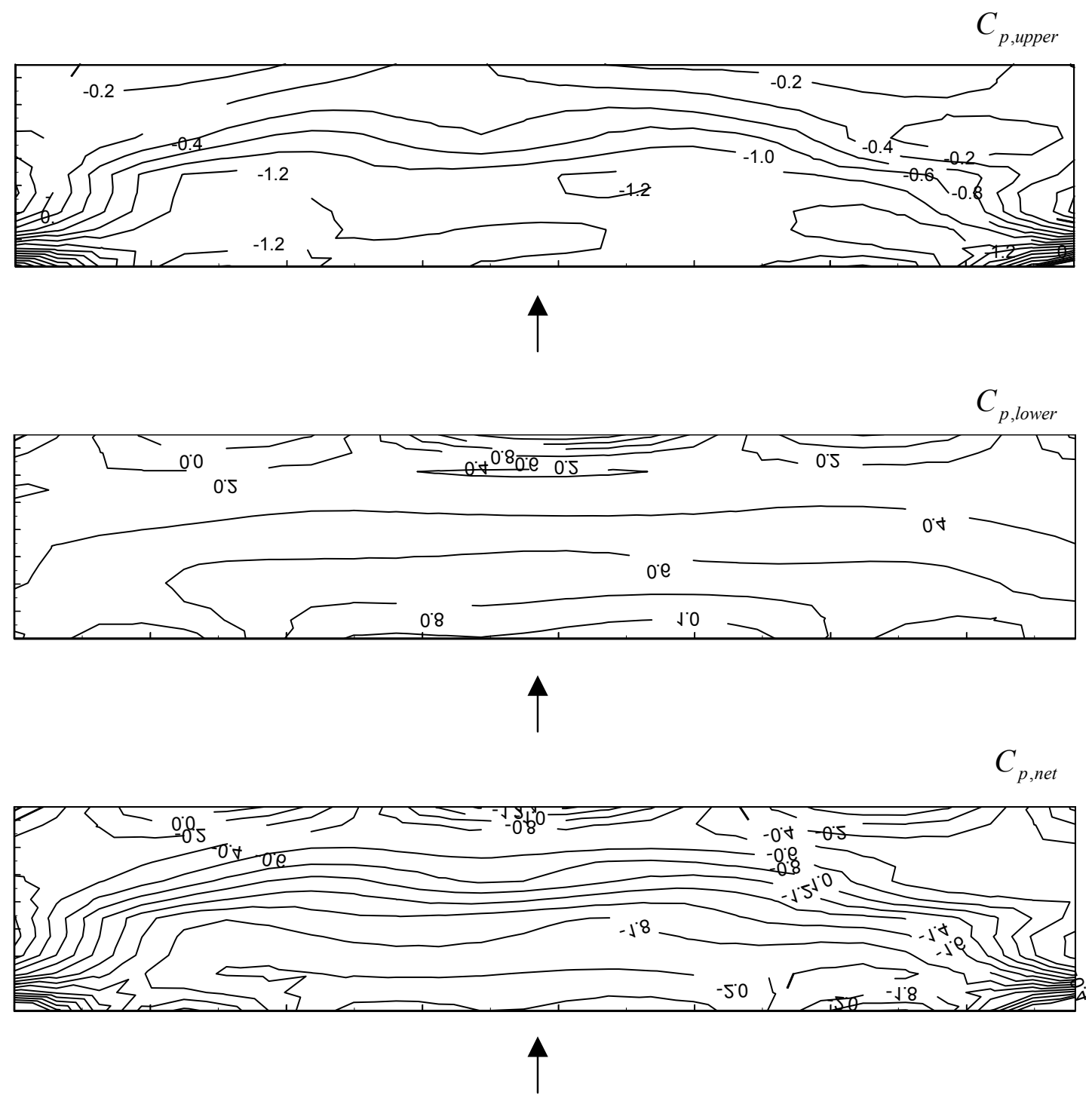

(a) $\alpha=0^{\circ}$

Fig. 14. Contour maps of pressure coefficients conditionally sampled at occurrences of peak total roof lift. Pressures shown for upper roof surface, lower roof surface and net roof pressure. $\alpha$ : (a) $0^{\circ}$; (b) $30^{\circ}$; (c) $60^{\circ}$; (d) $90^{\circ}$; (e) $120^{\circ}$; (f) $150^{\circ}$; (g) $180^{\circ}$. 

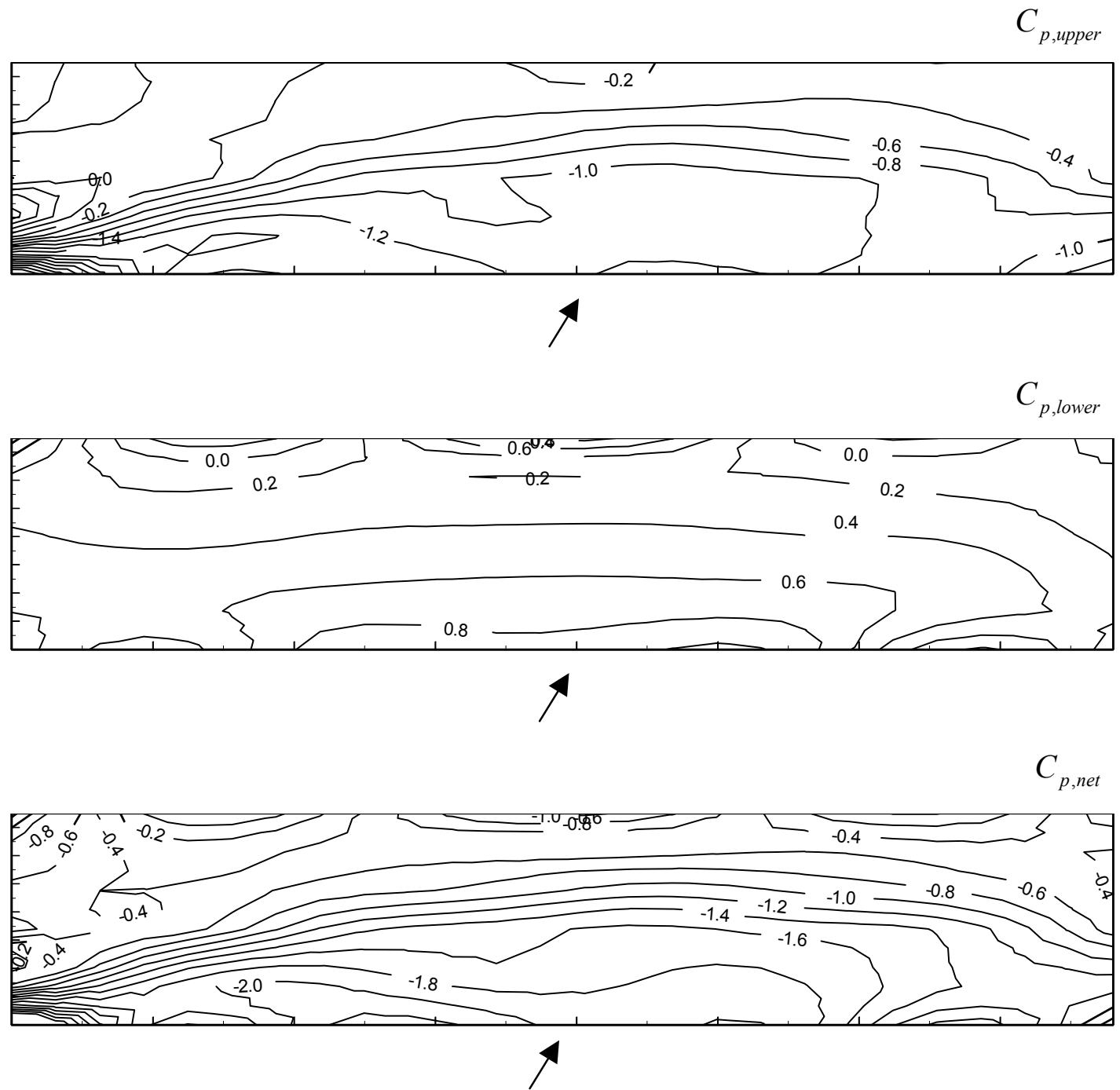

(b) $\alpha=30^{\circ}$

Fig. 14. Contour maps of pressure coefficients conditionally sampled at occurrences of peak total roof lift. Pressures shown for upper roof surface, lower roof surface and net roof pressure. $\alpha:$ (a) $0^{\circ}$; (b) $30^{\circ}$; (c) $60^{\circ}$; (d) $90^{\circ}$; (e) $120^{\circ}$; (f) $150^{\circ}$; (g) $180^{\circ}$. 

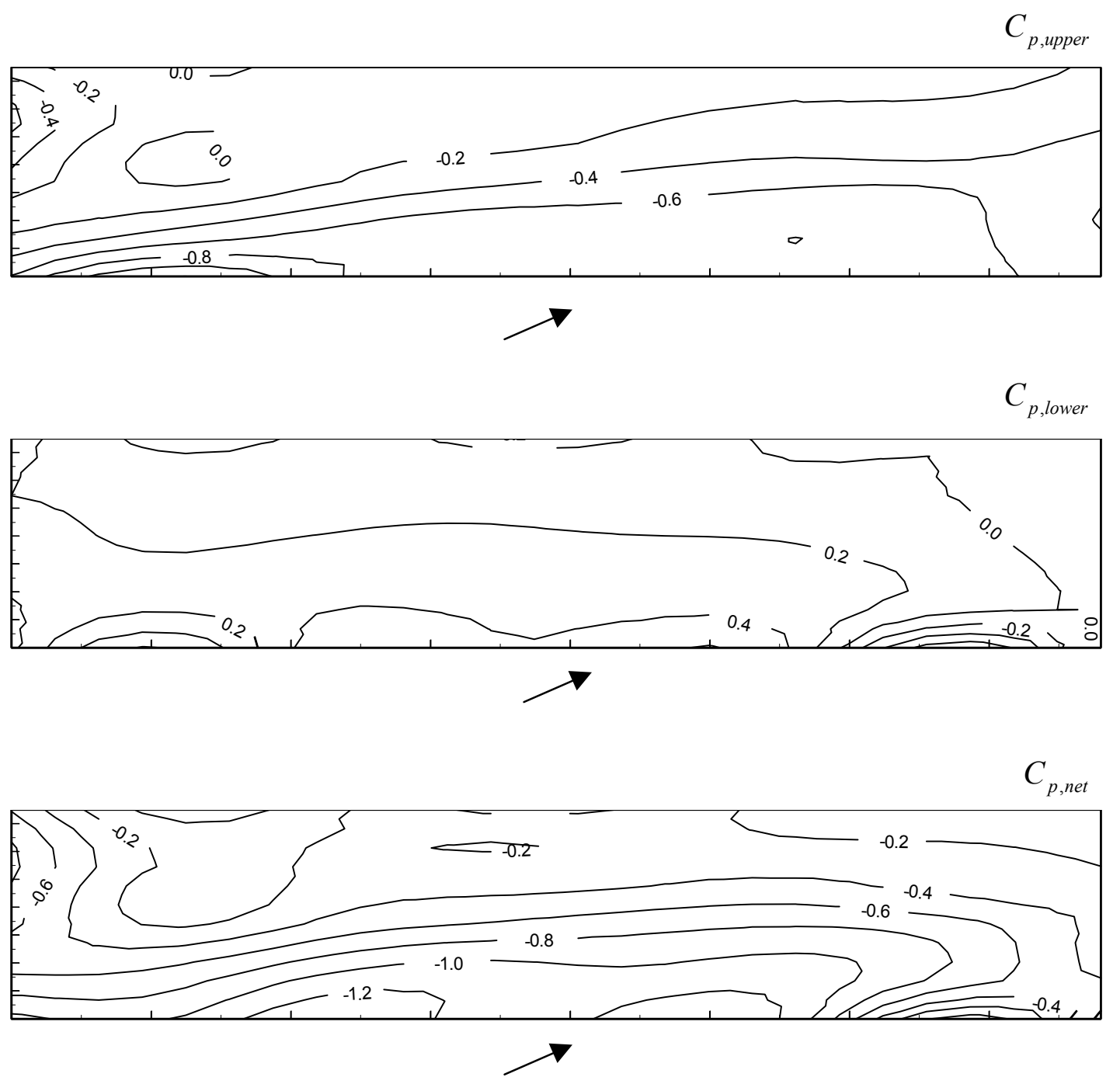

(c) $\alpha=60^{\circ}$

Fig. 14. Contour maps of pressure coefficients conditionally sampled at occurrences of peak total roof lift. Pressures shown for upper roof surface, lower roof surface and net roof pressure. $\alpha$ : (a) $0^{\circ}$; (b) $30^{\circ}$; (c) $60^{\circ}$; (d) $90^{\circ}$; (e) $120^{\circ}$; (f) $150^{\circ}$; (g) $180^{\circ}$. 
Lam \& Zhao, Fig. 14

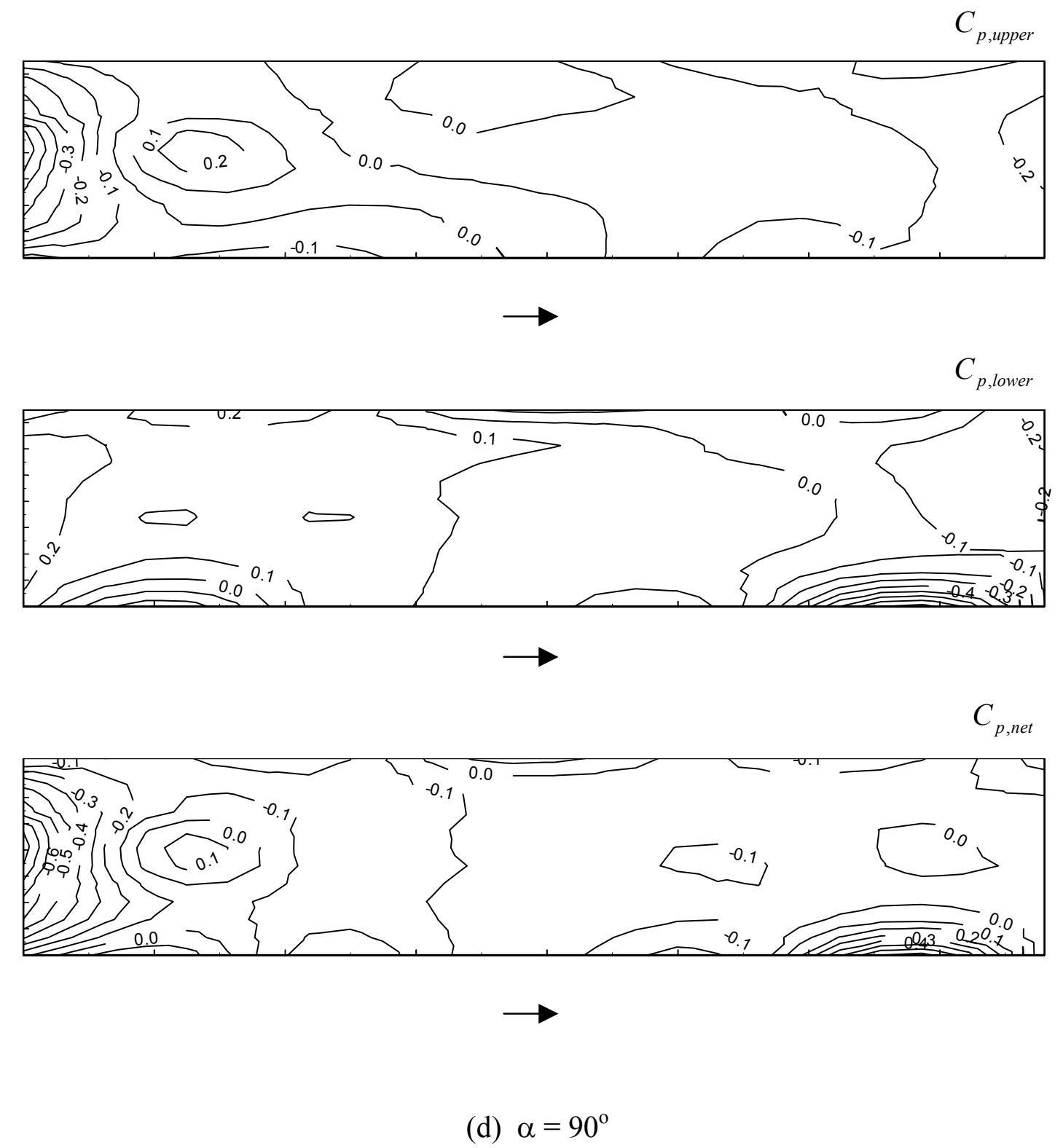

Fig. 14. Contour maps of pressure coefficients conditionally sampled at occurrences of peak total roof lift. Pressures shown for upper roof surface, lower roof surface and net roof pressure. $\alpha$ : (a) $0^{\circ}$; (b) $30^{\circ}$; (c) $60^{\circ}$; (d) $90^{\circ}$; (e) $120^{\circ}$; (f) $150^{\circ}$; (g) $180^{\circ}$. 

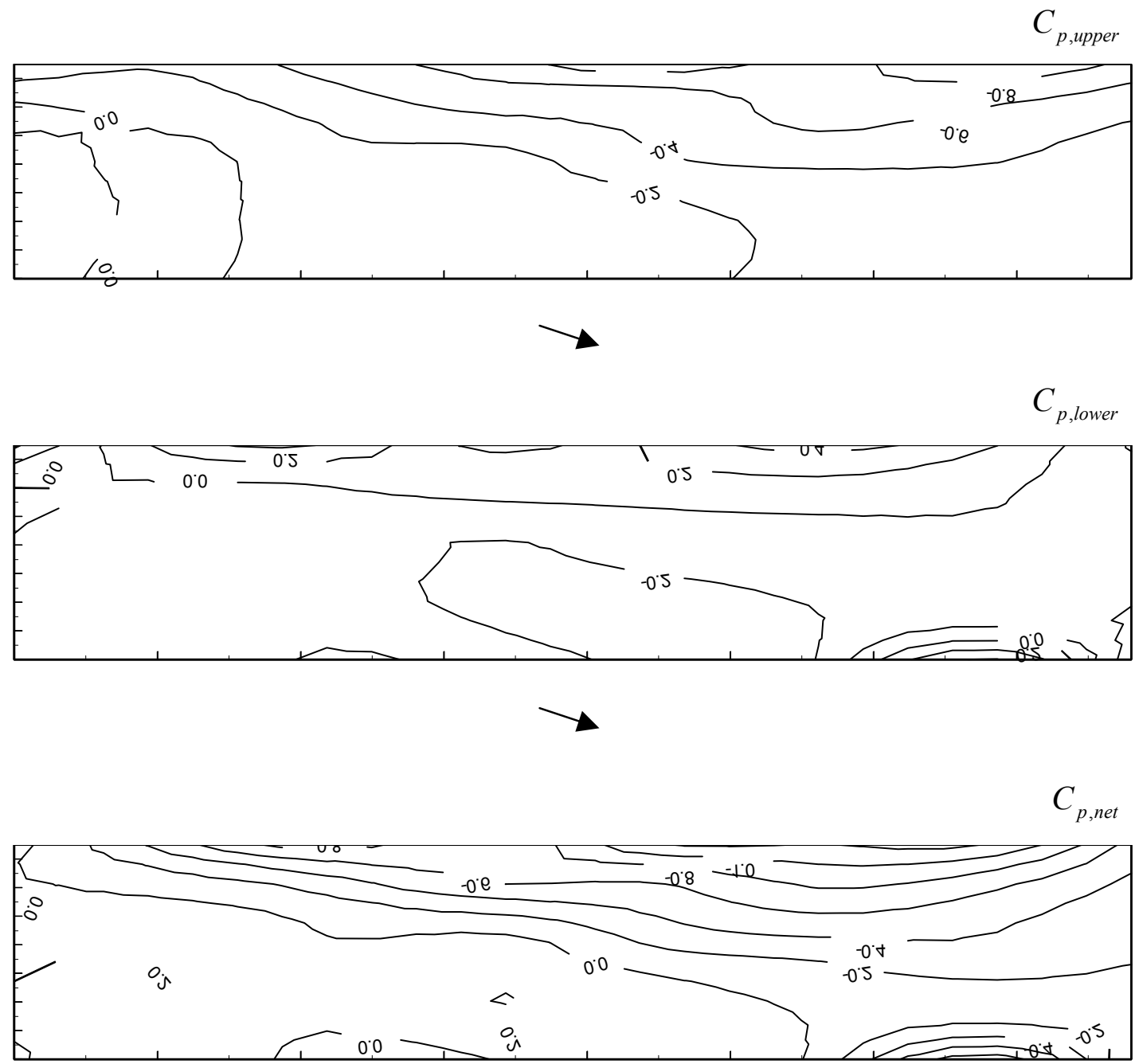

(e) $\alpha=120^{\circ}$

Fig. 14. Contour maps of pressure coefficients conditionally sampled at occurrences of peak total roof lift. Pressures shown for upper roof surface, lower roof surface and net roof pressure. $\alpha$ : (a) $0^{\circ}$; (b) $30^{\circ}$; (c) $60^{\circ}$; (d) $90^{\circ}$; (e) $120^{\circ}$; (f) $150^{\circ}$; (g) $180^{\circ}$. 

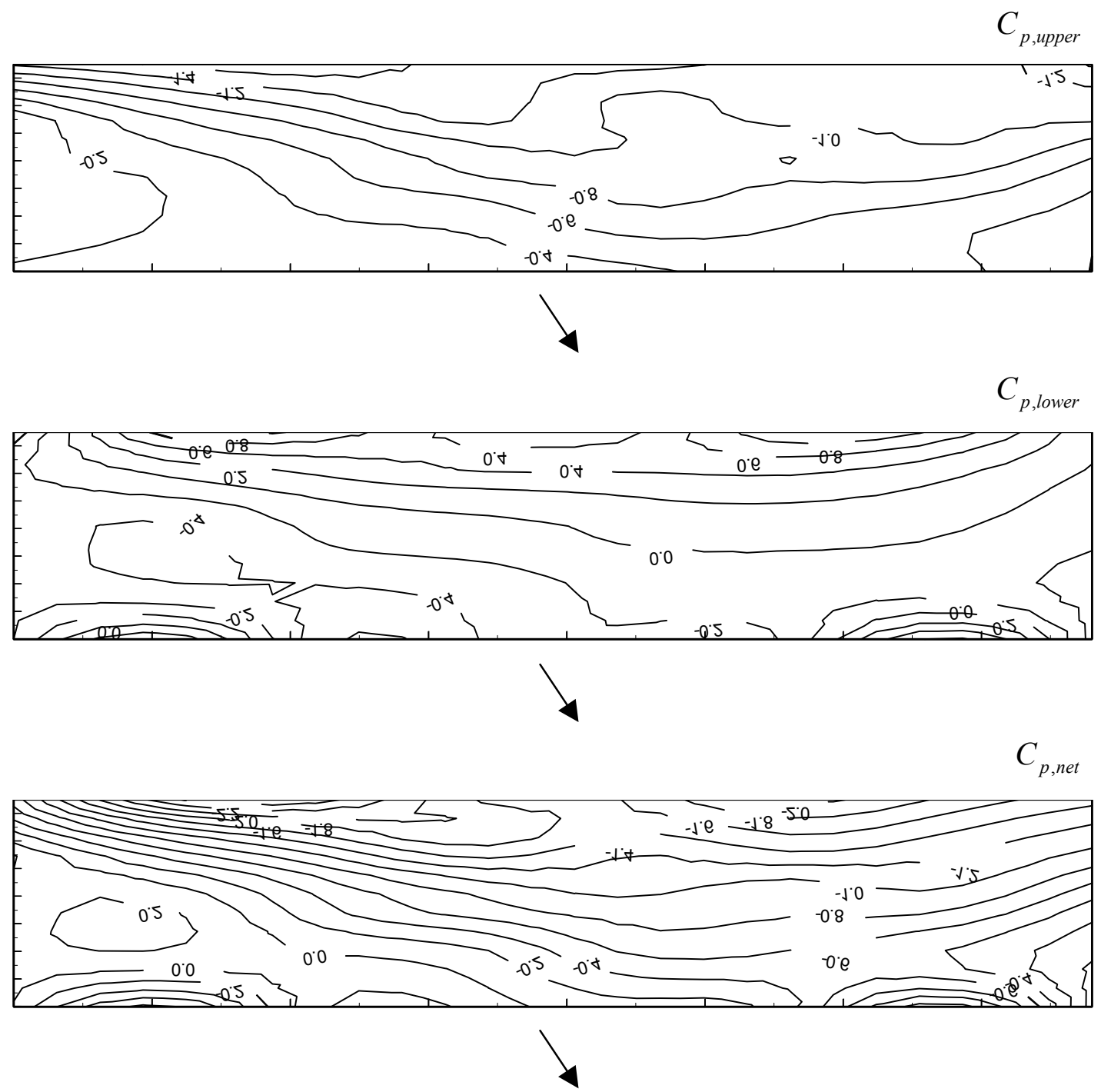

(f) $\alpha=150^{\circ}$

Fig. 14. Contour maps of pressure coefficients conditionally sampled at occurrences of peak total roof lift. Pressures shown for upper roof surface, lower roof surface and net roof pressure. $\alpha$ : (a) $0^{\circ}$; (b) $30^{\circ}$; (c) $60^{\circ}$; (d) $90^{\circ}$; (e) $120^{\circ}$; (f) $150^{\circ}$; (g) $180^{\circ}$. 


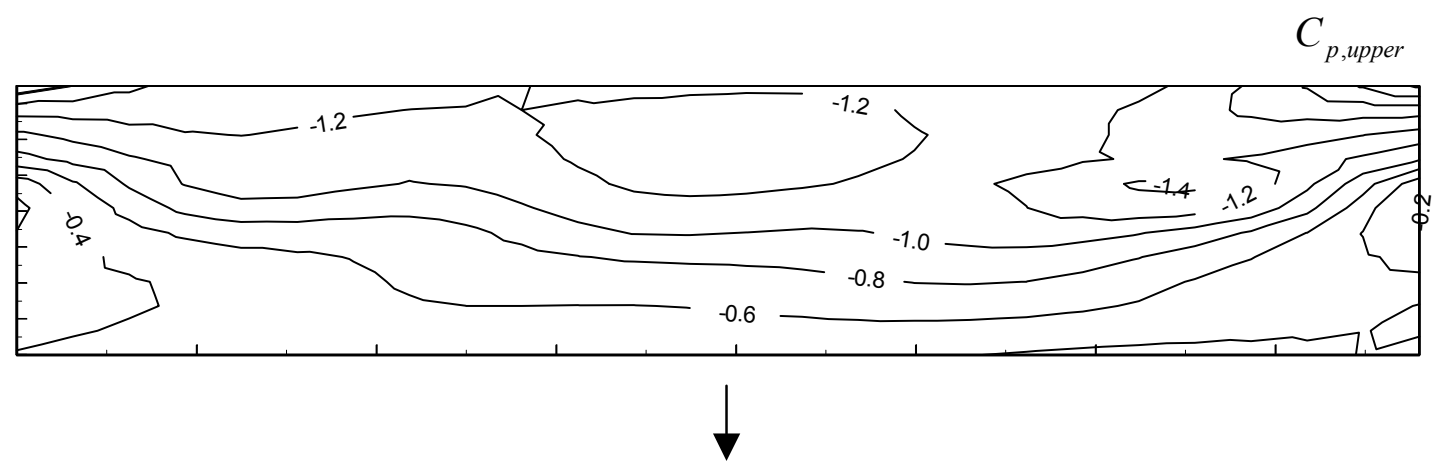

$C_{p, l o w e r}$
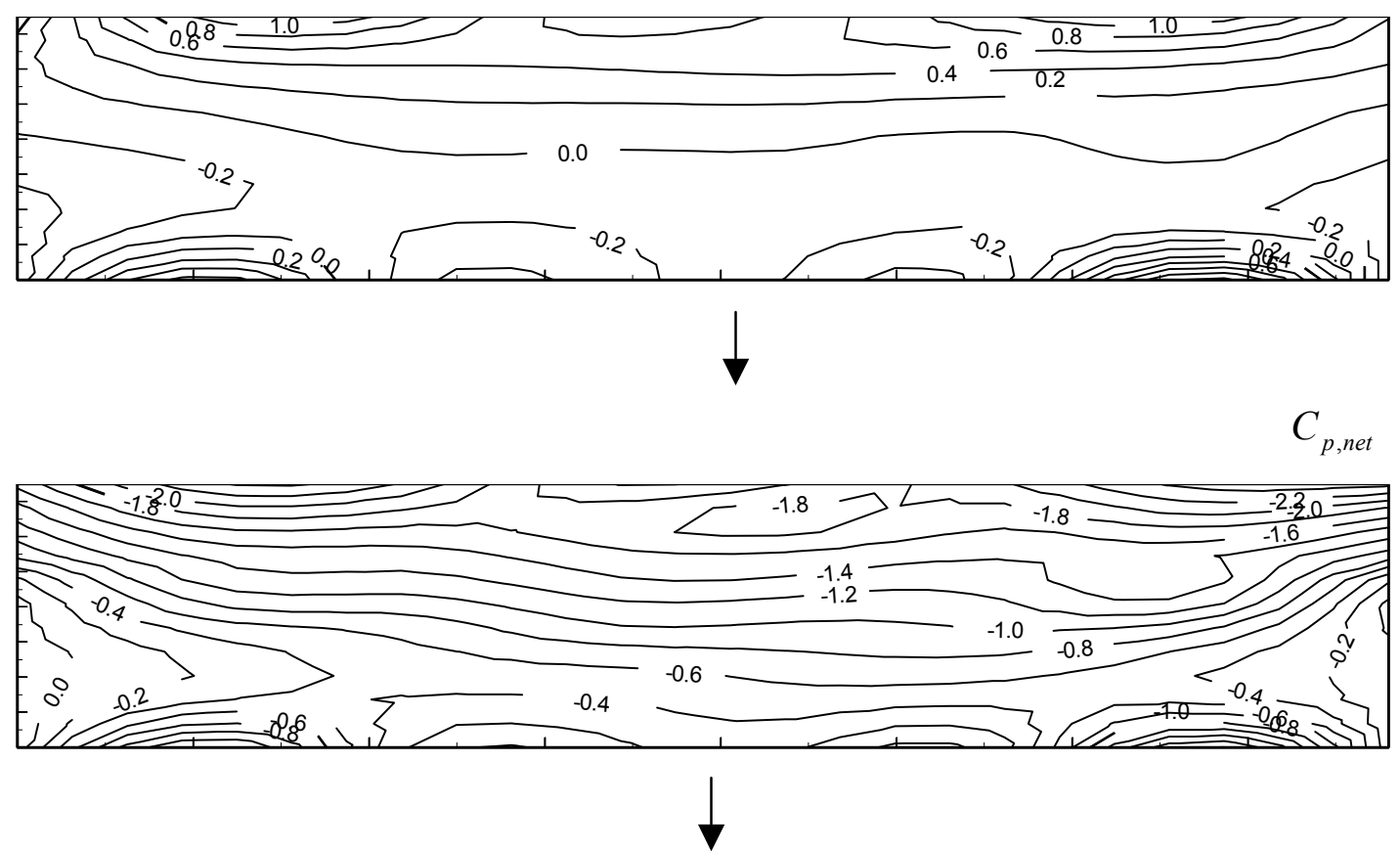

(g) $\alpha=180^{\circ}$

Fig. 14. Contour maps of pressure coefficients conditionally sampled at occurrences of peak total roof lift. Pressures shown for upper roof surface, lower roof surface and net roof pressure. $\alpha$ : (a) $0^{\circ}$; (b) $30^{\circ}$; (c) $60^{\circ}$; (d) $90^{\circ}$; (e) $120^{\circ}$; (f) $150^{\circ}$; (g) $180^{\circ}$. 

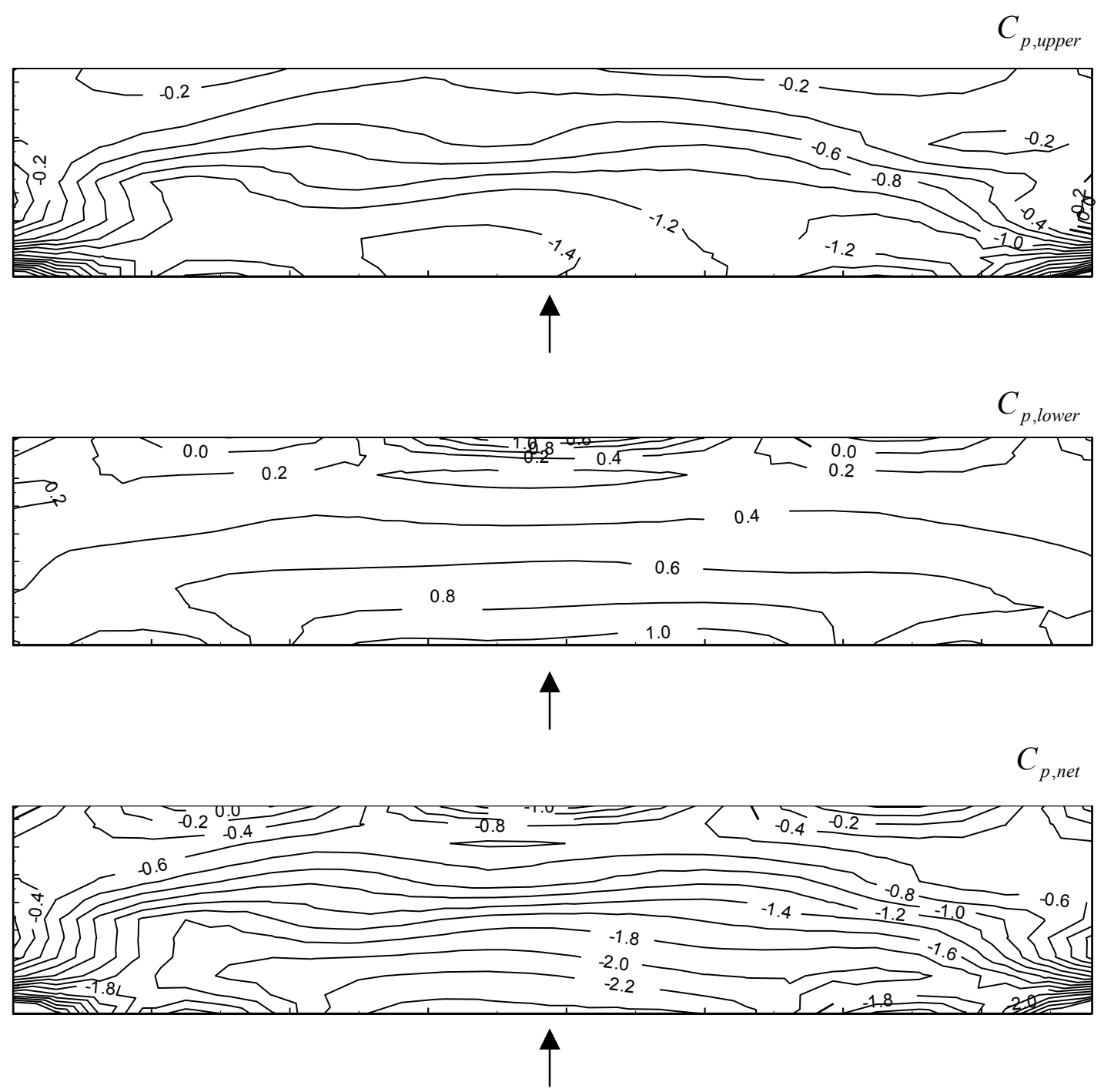

(a) $\alpha=0^{\circ}$

Fig. 15. Distribution of pressure coefficients conditionally sampled at occurrences of peak total roof moment. Pressures shown for upper roof surface, lower roof surface and net roof pressure.

$$
\alpha \text { : (a) } 0^{\circ} \text {; (b) } 30^{\circ} \text {; (c) } 60^{\circ} \text {; (d) } 90^{\circ} \text {. }
$$



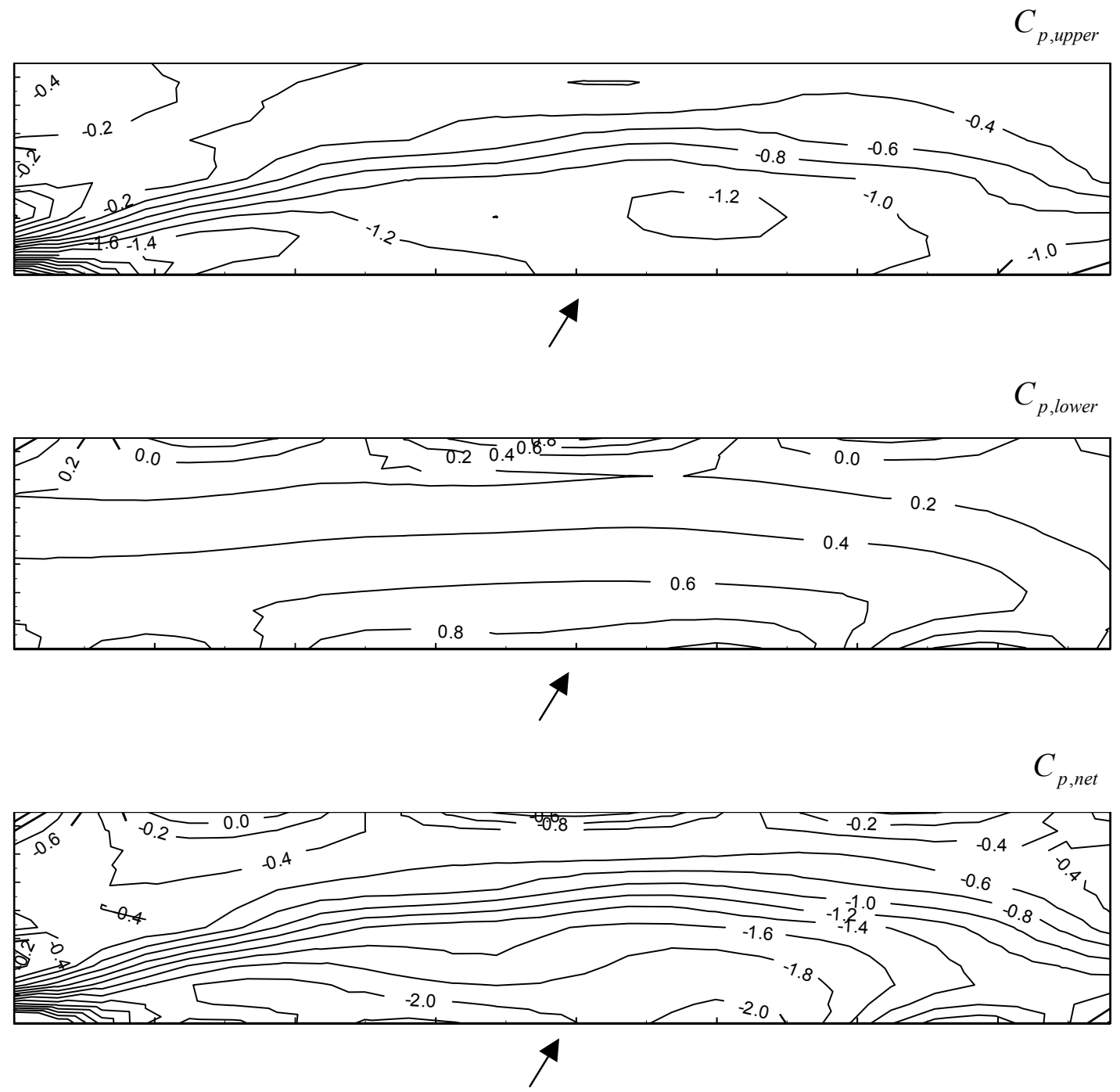

(b) $\alpha=30^{\circ}$

Fig. 15. Distribution of pressure coefficients conditionally sampled at occurrences of peak total roof moment. Pressures shown for upper roof surface, lower roof surface and net roof pressure.

$$
\alpha \text { : (a) } 0^{\circ} \text {; (b) } 30^{\circ} \text {; (c) } 60^{\circ} \text {; (d) } 90^{\circ} \text {. }
$$



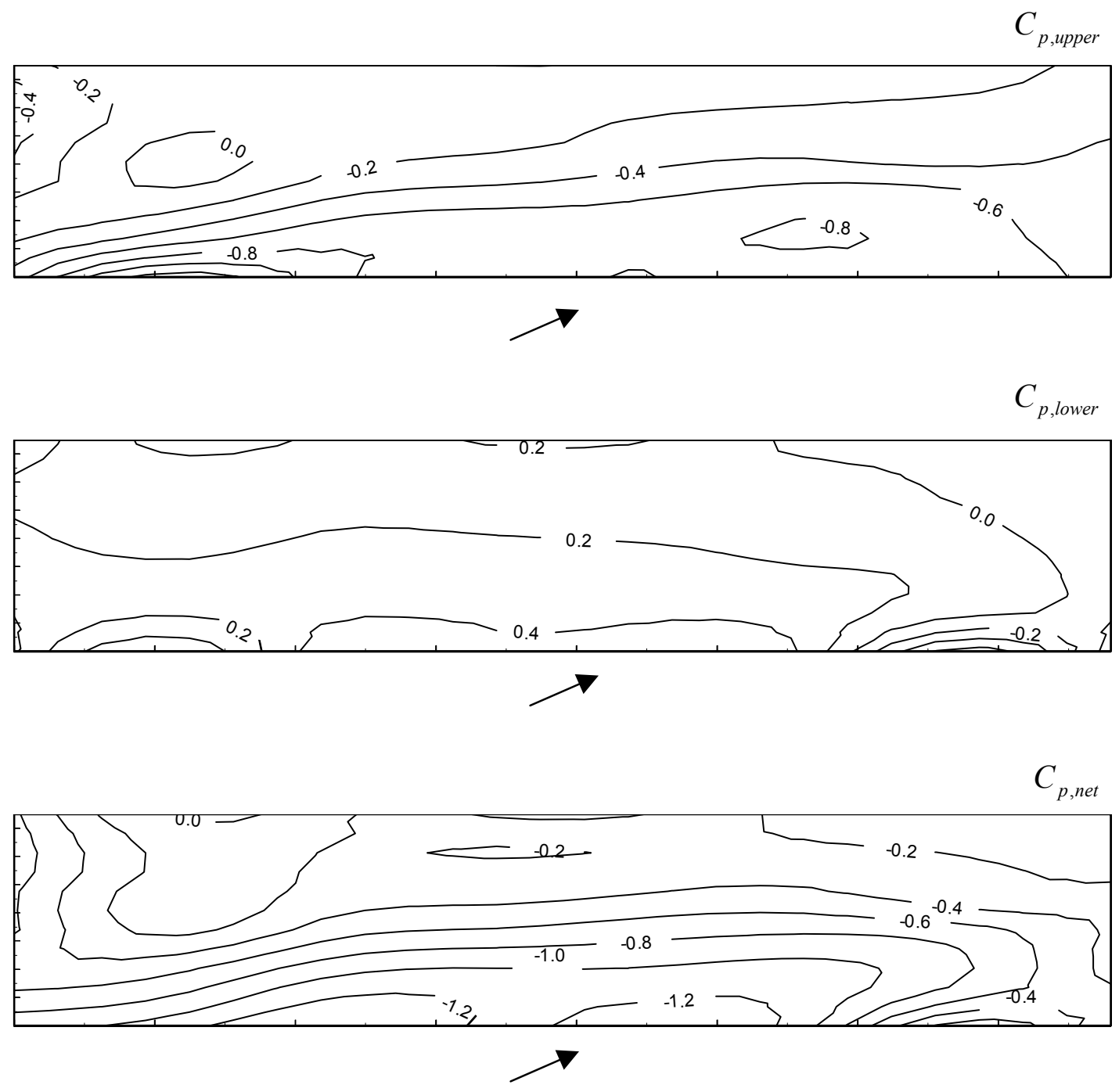

(c) $\alpha=60^{\circ}$

Fig. 15. Distribution of pressure coefficients conditionally sampled at occurrences of peak total roof moment. Pressures shown for upper roof surface, lower roof surface and net roof pressure. $\alpha:$ (a) $0^{\circ}$; (b) $30^{\circ}$; (c) $60^{\circ}$; (d) $90^{\circ}$. 
Lam \& Zhao, Fig. 15

$C_{p, \text { upper }}$
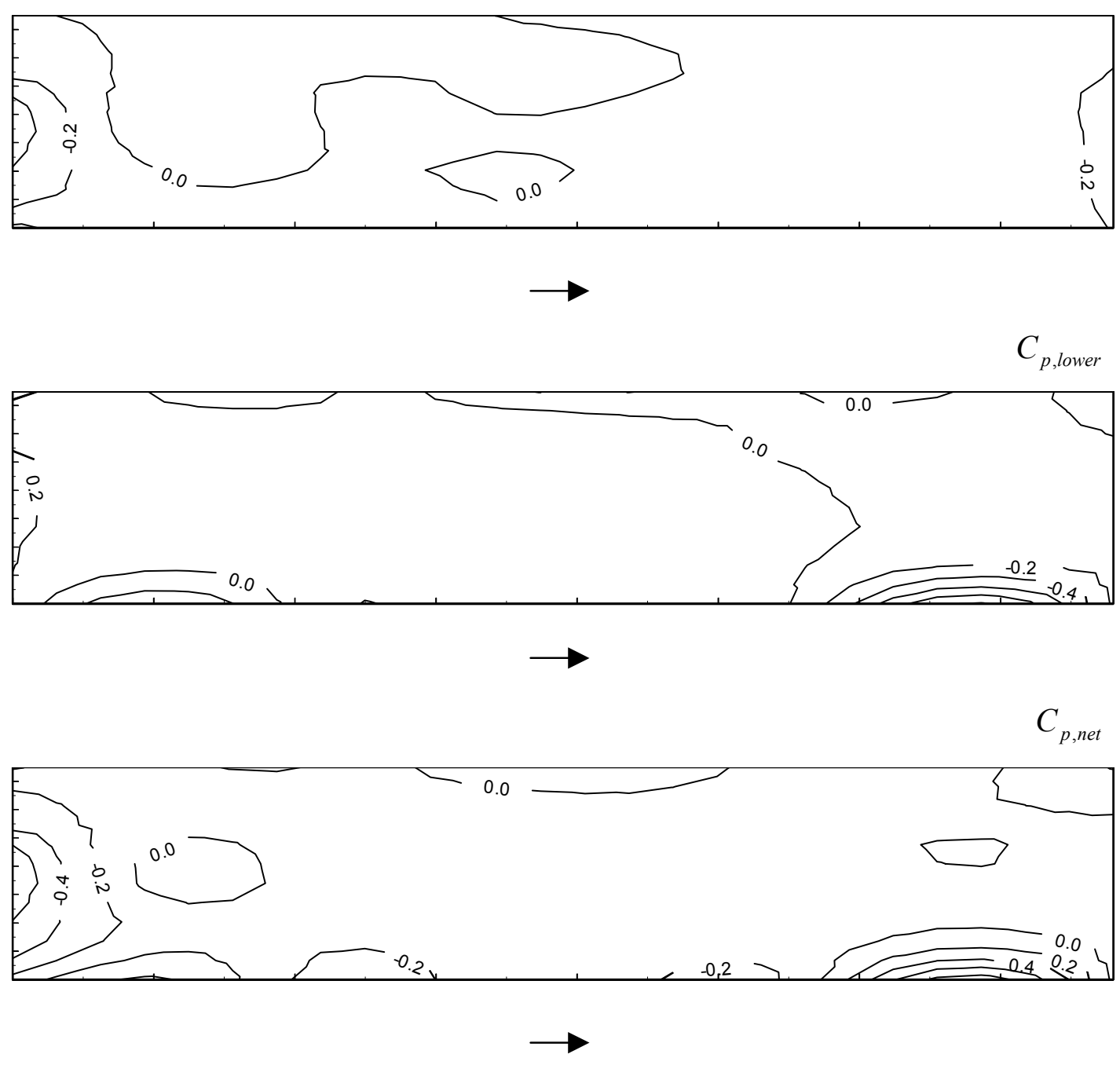

(d) $\alpha=90^{\circ}$

Fig. 15. Distribution of pressure coefficients conditionally sampled at occurrences of peak total roof moment. Pressures shown for upper roof surface, lower roof surface and net roof pressure. $\alpha$ : (a) $0^{\circ}$; (b) $30^{\circ}$; (c) $60^{\circ}$; (d) $90^{\circ}$. 


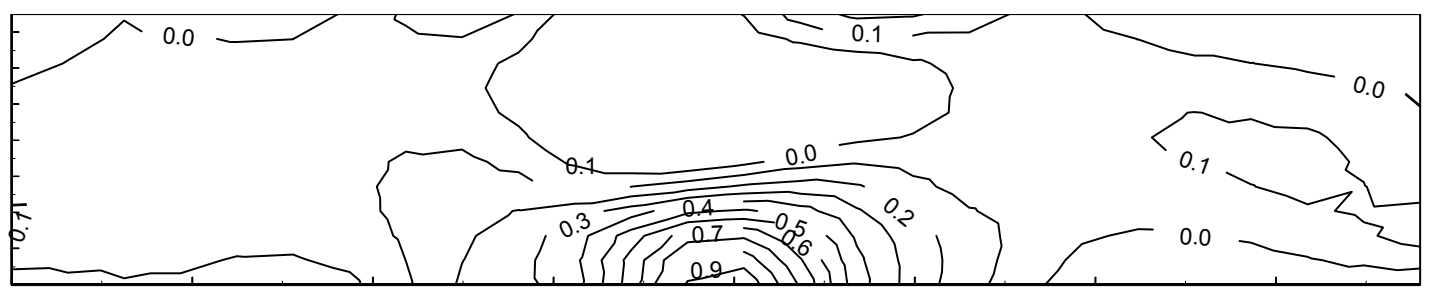

$$
\uparrow \alpha=0^{\circ}
$$

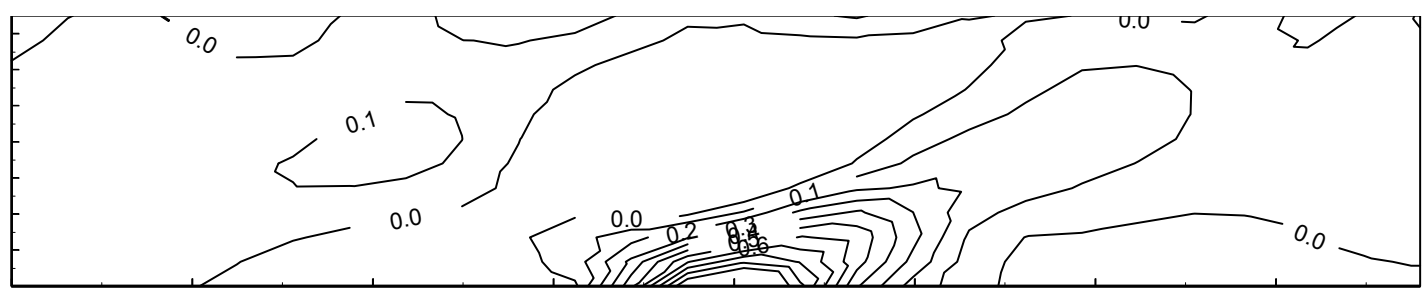

$$
\alpha=30^{\circ}
$$

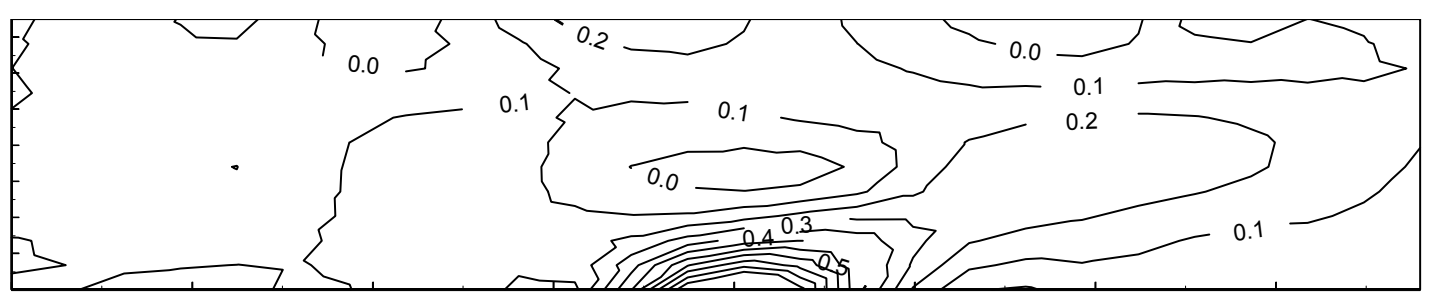

$\gamma \alpha=60^{\circ}$

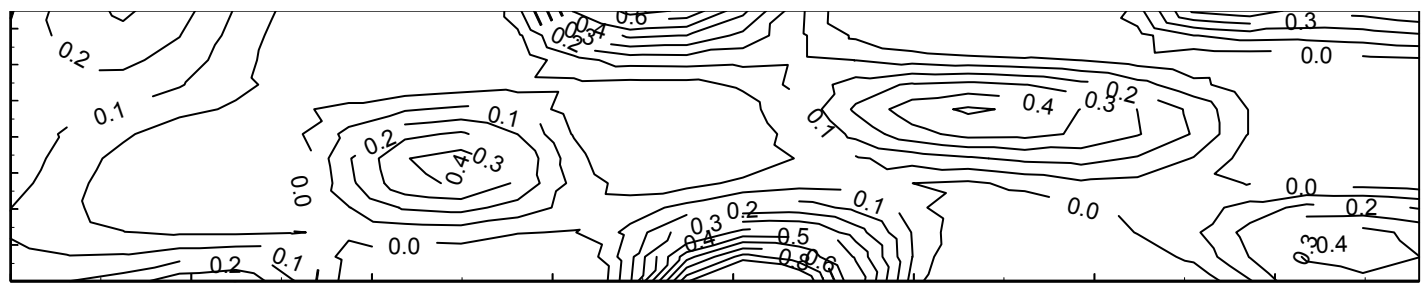

$\rightarrow \alpha=90^{\circ}$

Fig. 16. Cross-correlation coefficients between net pressure on roof surface and reference net pressure at most front tap location at mid-span. $\alpha$ : (a) $0^{\circ}$; (b) $30^{\circ}$; (c) $0^{\circ}$; (d) $90^{\circ}$. 

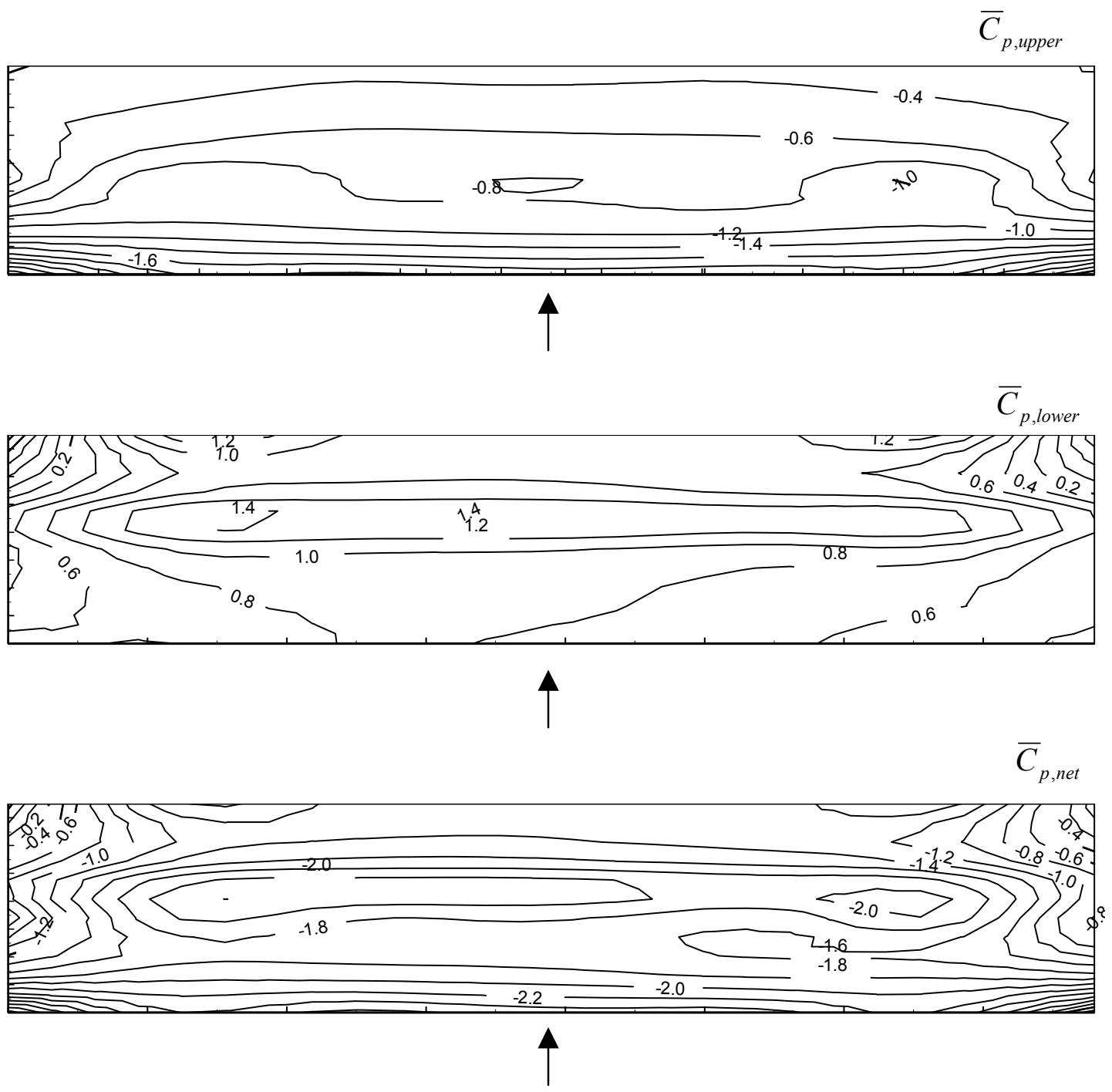

(a) $100 \%$ grandstand blockage

Fig. 17. Contours of mean pressure coefficients on upper roof surface, lower roof surface, and net roof pressure at $\alpha=0^{\circ}$. Grandstand blockage: (a) $100 \%$; (b) 0 . 

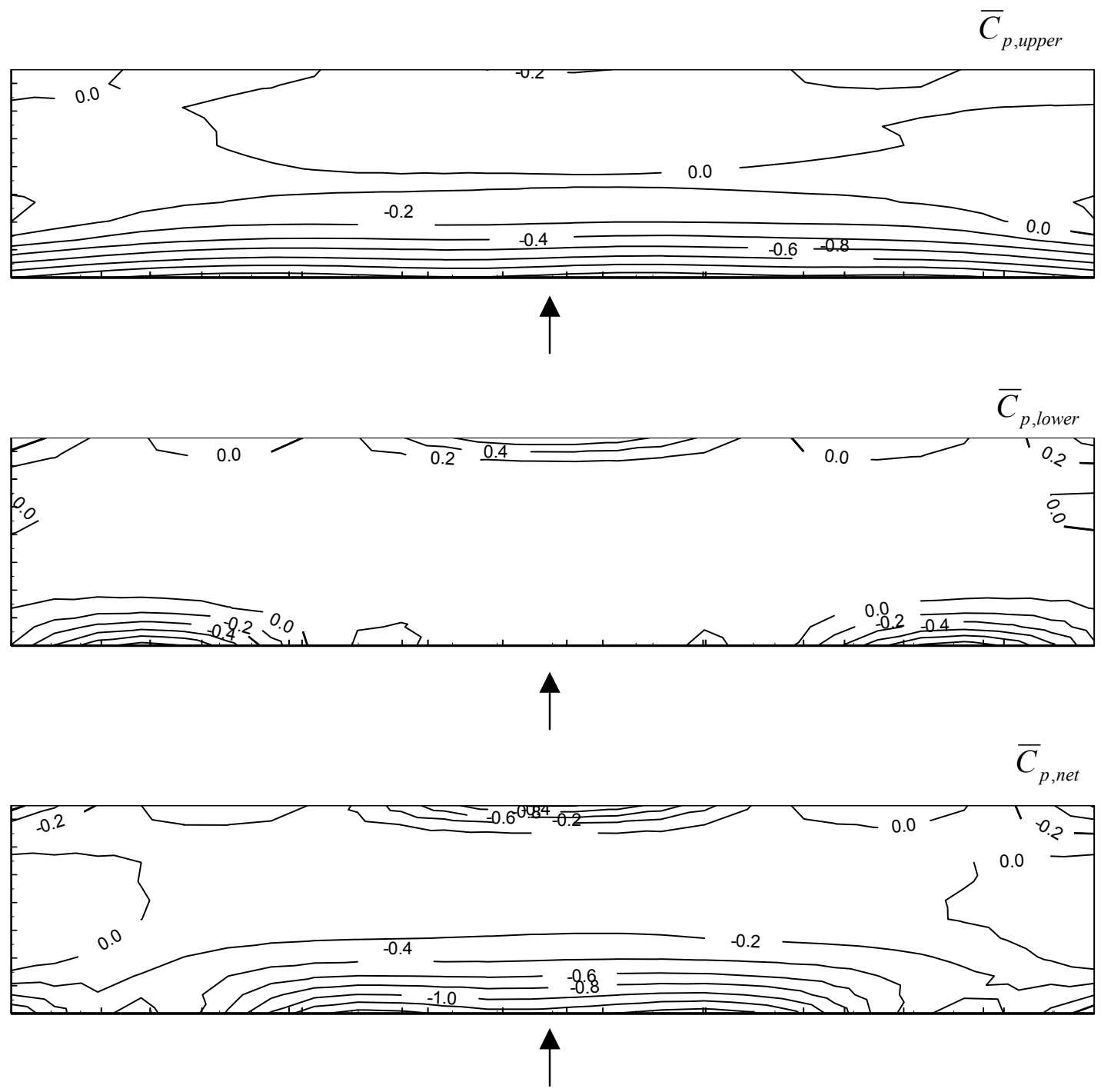

(b) No grandstand blockage

Fig. 17. Contours of mean pressure coefficients on upper roof surface, lower roof surface, and net roof pressure at $\alpha=0^{\circ}$. Grandstand blockage: (a) $100 \%$; (b) 0 . 